\title{
Polycyclic aromatic hydrocarbons in post-fire soils of drained peatlands in western Meshchera (Moscow region, Russia)
}

\author{
A. Tsibart $^{1}$, A. Gennadiev ${ }^{1}$, T. Koshovskii ${ }^{1}$, and A. Watts ${ }^{2}$ \\ ${ }^{1}$ Moscow State University, Faculty of Geography, Department of Landscape Geochemistry and Soil Geography, \\ 119991, Leninskie gori, 1, Moscow, Russia \\ ${ }^{2}$ Desert Research Institute, Division of Atmospheric Sciences, 2215 Raggio Parkway, Reno, NV 89512, USA \\ Correspondence to: A. Tsibart (tsibann@gmail.com) and A. Gennadiev (alexagenna@mail.ru)
}

Received: 28 March 2014 - Published in Solid Earth Discuss.: 16 May 2014

Revised: 10 November 2014 - Accepted: 11 November 2014 - Published: 16 December 2014

\begin{abstract}
Polycyclic aromatic hydrocarbons (PAHs) are priority pollutants that arrive in the environment from numerous anthropogenic and natural sources, but the data on their natural sources including wildfires remain insufficient. The level of contamination and the composition of PAHs in soils of the areas affected by wildfires were studied in this work. The study was conducted in the Moscow region (Russia) in areas occupied by drained peatland and strongly damaged by fires in 2002, 2010 and 2012. The features of PAH accumulation and the profile distributions in histosols and histic podzols after the fires of different times were analyzed. It was shown that new soil horizons formed after the fires - Cpir, Hpir and incipient $\mathrm{O}$ horizons - and that these horizons differ in PAH accumulation rate. Maximal total concentrations of 14 PAHs were detected in charred peat horizons Hpir (up to $330 \mathrm{ng} \mathrm{g}^{-1}$ ) and in postfire incipient $\mathrm{O}$ horizons (up to $180 \mathrm{ng} \mathrm{g}^{-1}$ ), but the highmolecular-weight PAHs (benz(ghi)perylene, benz(a)pyrene, benz(k)fluoranthene) were revealed only in charry peat horizons. The trends of higher PAH concentrations were found in cases when smoldering combustion resulted in rather thick residual peat horizons. In cases of almost complete pyrogenic destruction of He horizons, total PAH concentrations were no more than $50 \mathrm{ng} \mathrm{g}^{-1}$. Also, PAH accumulation in upper horizons of soils near the sites of the latest fires was observed.
\end{abstract}

\section{Introduction}

Polycyclic aromatic hydrocarbons (PAHs) are a group of high molecular weight organic compounds, which include carcinogens and mutagens. PAHs are formed in natural and technogenic processes, and are ubiquitous in different landscape components. Scientific interest in PAHs has remained high during recent decades (Wilcke, 2000; Haritash and Kaushik, 2009; Cai et al., 2008; Gennadiev et al.,1996; Bandowe et al., 2014, Maisto et al., 2006, Baek et al., 1991). Most PAHs in the environment arrive from pyrogenic sources. So far, pyrogenic anthropogenic sources of PAHs (automobile exhaust, various industrial and power plant emissions) are well studied (Khan et al., 2008; Mastral and Callen, 2000; Tsibart and Gennadiev, 2013; Wilcke, 2000, 2007; Agarwal, 2009; Kwon, 2014; Mu et al., 2013) and, nowadays, in developed countries, there is a trend of reducing environmental contamination with PAHs because of improvement in technologies (Guo et al., 2011).

The number of PAHs arriving in the environment from natural pyrogenic sources (wildfires, volcanism) remains uncertain. Numerous works are devoted to PAH formation from vegetation components influenced by flaming and smoldering combustion. Burning conditions (the amount of available oxygen, the duration of heating, the temperature) and the type of vegetation define the amount and composition of PAHs (Ramdahl and Bechler, 1982; Jenkins, 1996; Nussbaumer, 2003; Medeiros and Simoneit, 2008; Simoneit, 1999; Schauer et al., 2001; Nakajima et al., 2007; Fitzpatrick et al., 2008; Kakareka et al., 2004). 
The publications devoted to pyrogenic PAHs in the territories affected by wildfires do not cover the range of questions related to this problem. For instance, the peculiarities of PAH composition in the air after a wildfire were studied in several works (Radojevic, 2003; Masclet et al., 1995, Maioli et al., 2009; Freeman and Cattell, 1990; Yuan et al., 2008), but there is an obvious lack of information on pyrogenic PAH accumulation in soils. Some publications are focused on PAHs in different organo-mineral soils that were subjected to fire (Gennadiev and Tsibart, 2013; Dymov et al., 2014; Gonzalez-Vila et al., 1991; Garcia-Falcoan et al., 2006; Vergnoux et al., 2011). It was shown that PAHs accumulated in these soils in small amounts and posed no danger to humans. However, PAH accumulation in soils after smoldering fires causing deep changes in soil profiles was investigated insufficiently (Vane et al., 2013; Gennadiev and Tsibart, 2013; Bojakowska and Sokołowska, 2003).

Peat fires differ from other fire types, because the burning material in this case is not only the vegetation, but also the soil organic matter. The conditions present in peat smoldering favor PAH formation because fires propagate slowly and deep soil horizons are affected by high temperature (Rein et al., 2008; Hartford and Frandsen, 1992). Moreover, in comparison to flaming fires, smoldering consumes most of the peat. Also, smoldering drives the spread of the pyrolysis front where PAHs are produced, and the smoldering process occurs under conditions of low oxygen and temperature (500$700^{\circ} \mathrm{C}$ ) compared with flaming fire (Rein, 2013). These conditions favor the processes of organic radical recombination and PAH formation.

It is worth noting that the combustion products differ depending on the peat type, moisture and the completeness of combustion. Products of pyrolysis are a gaseous mixture of organic species released into the air. They include volatile organic compounds, hydrocarbons $\left(\mathrm{CH}_{4}, \mathrm{C}_{3} \mathrm{H}_{8}, \mathrm{CH}_{3} \mathrm{OH}\right)$ and particulate matter. During the oxidation stage of combustion, $\mathrm{CO}$ and $\mathrm{CO}_{2}$ are produced (Rein, 2013). Peat smoke also contains sulfur, potassium, oxides of nitrogen (Blake et al., 2009) and metals ( $\mathrm{Al}, \mathrm{Cd}, \mathrm{Co}, \mathrm{Cr}, \mathrm{Cu}, \mathrm{Fe}$, Mn, Ni, Pb, Ti, V, Zn) (Betha et al., 2013). PAHs are part of the peat combustion products in most cases and, after the fires, they generally accumulate across the whole soil profile (Vane et al., 2013; Kakareka et al., 2004; Blake et al., 2009). So, PAHs can accumulate in situ in char and ash layers before they are released into the atmosphere (Rein, 2013). Moreover, below the fire front, higher production of aromatic and condensed molecules takes place (Zaccone et al., 2014), and gaseous PAHs after releasing into the atmosphere can also be transported and accumulate at other sites (Rein, 2013).

In most cases, the scientific literature contains data on pyrogenic PAHs in undrained peat soils. The drained soils, following fire, are not studied from this context, although they are very vulnerable to wildfires (Blake et al., 2009; Zaidel'man et al., 2007). Large amounts of organic matter burn out during the fires, and deep transformations occur in the profiles, in comparison with the soils of undrained territories (Zaidel'man et al., 2007, 1999). The drained peat soils are widespread in densely populated areas of the European part of Russia, and an important task is to reveal levels of accumulation of PAHs in these areas.

The aim of this study was to reveal the features of PAH accumulation in podzols and histosols of drained peatlands affected by wildfires. Study objectives included (1) the comparison of PAH distributions and levels of accumulation in different post-fire soils, histosols and podzols, (2) the identification of the parts of post-fire soil profiles with maximal PAH accumulation, (3) the detection of the trends of different PAH group accumulation, and (4) the comparison of PAH accumulation in soils after fires of different times.

\section{Materials and methods}

\subsection{Study area}

This study was conducted in the soils of Shatura district (Moscow region, Russia). The burned area is located at coordinates $55^{\circ} 39^{\prime} \mathrm{N}, 39^{\circ} 36^{\prime} \mathrm{E}$ with an elevation of $120 \mathrm{~m}$. The investigated site is situated $15 \mathrm{~km}$ to the north of Shatura (Fig. 1) and $200 \mathrm{~m}$ to the west of the village of Dolgusha (Fig. 2).

The territory represents the western Meshchera fluvioglacial plain. The major part of the area is covered by fluvial-glacial deposits, although alluvial deposits also occur. The elevation is within the limits of 120-126 m, and the relief is low (Wetlands of Moscow Region, 2008; Zonov and Konstantinovich, 1932).

The investigated area is covered with peatlands overlying ancient alluvial deposits, which are located above the confining clay layer. The development of these wetlands is caused by a flattened relief and a shallow horizon of waterproof clay. The area covered with peat bogs in this part of the Meshchera plain is $15-16 \%$ (Wetlands of Moscow Region, 2008; Zonov and Konstantinovich, 1932; Kudravtseva, 1973).

The investigated plot belongs to the PetrovskoKobelevskoe high-moor peat, which is a part of the Shatura wetland area. Its total area is 6443 ha; before the era of peat extraction, the thickness of the peat layer reached $7.5 \mathrm{~m}$, and its average thickness was $2.5-4 \mathrm{~m}$ (Zonov and Konstantinovich, 1932).

These large areas were transformed during the drainage and peat mining. Beginning at the end of the eighteenth century, the peat bogs of the Moscow region were used as peat fields. The demand for peat increased in the 1920s, when peat-burning power stations including Shaturskaya station were constructed (Wetlands of Moscow Region, 2008; Simakin, 1958). The peat deposits in the Shatura district were mined beginning in the 1920s (Timashev, 1932). Now mining no longer occurs, and most of the peat bogs in Meshchera 

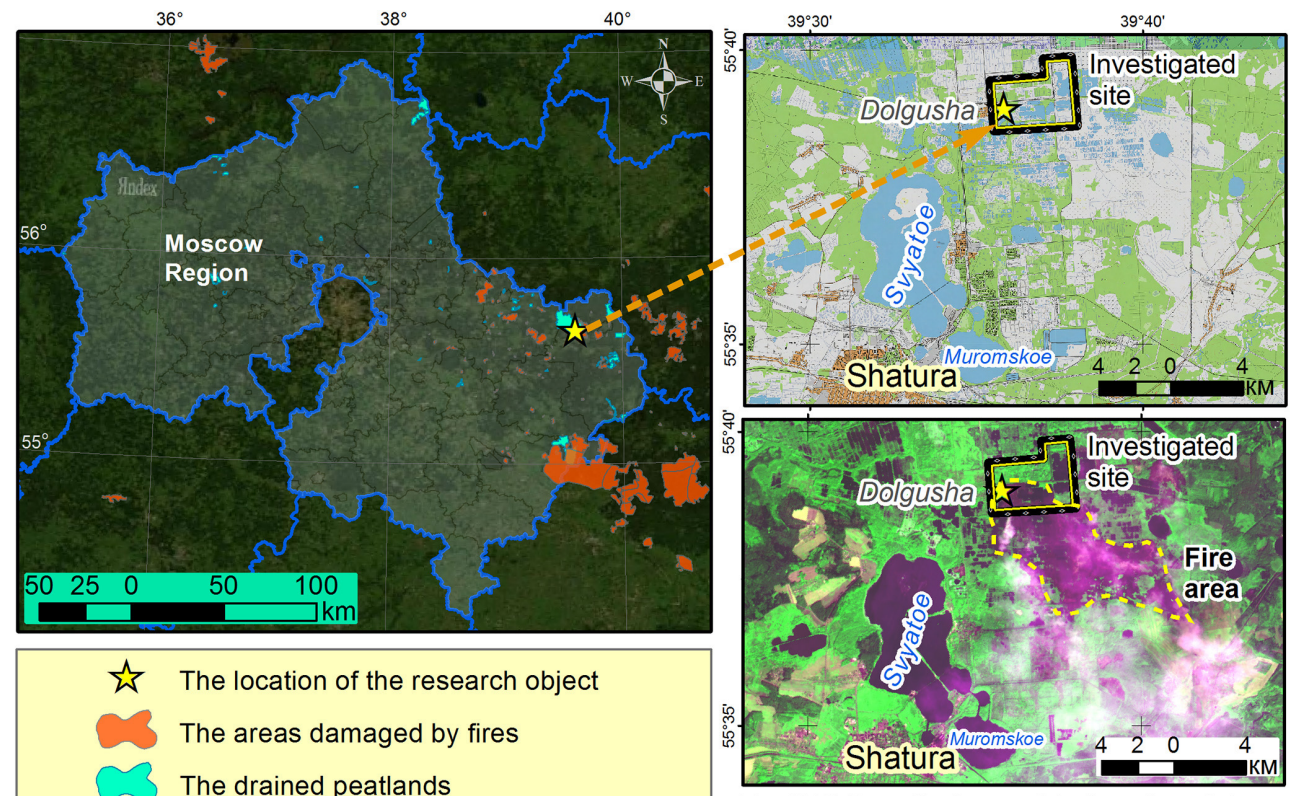

The SPOT4 satellite image of the investigated site provided by Geoportal MSU (12 August 2010, the moment of the wildfire).

Figure 1. The location of the research site.

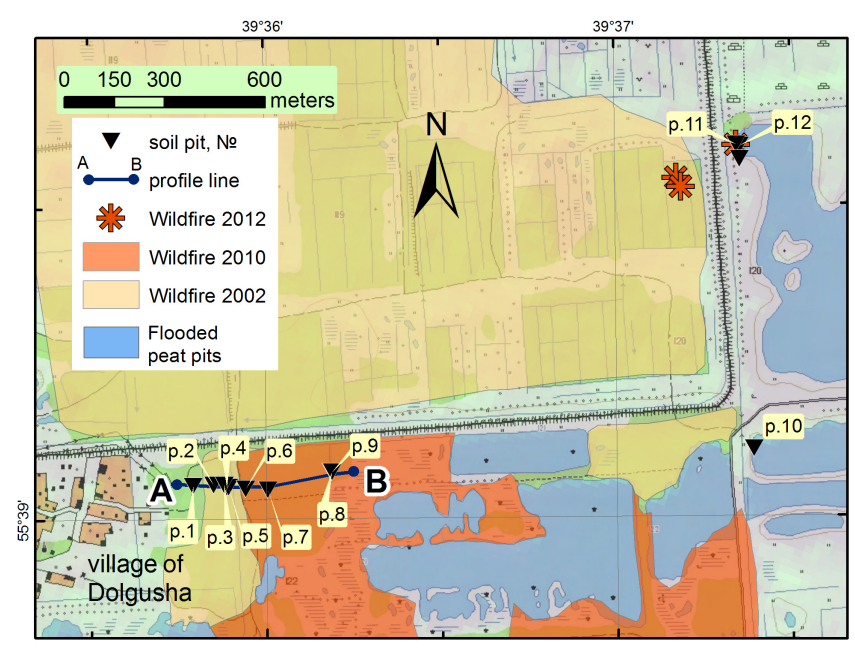

Figure 2. The location of the investigated soil pits.

bog province are in a stage of recovery (Wetlands of Moscow Region, 2008).

As a result of peat mining, natural bog complexes were changed to large open pits connected to systems of channels and distributaries and to fields of peat mining at different stages of recovery, with birch-aspen forests occurring on their banks. On the plots with flooded peat pits, the process of bog restoration has started, but it will take several centuries before peat deposits will start to accumulate (Sushkova, 2008).
Because of changes in hydrological regime, the number of areas of peat fires has increased dramatically in this region (Wetlands of Moscow Region, 2008). According to Zaidel'man (2003), there is a repeating pattern of the fires in drained peatlands. Almost every 10 years, large fires occur (1972, 1982, 1992, 2002). In 2010, wildfires in Russia damaged large areas and, in the Moscow region, they caused significant air pollution, degradation of ecosystems and health impacts for the population (Donkelaar et al., 2011; Shvidenko et al., 2011).

The soil cover of the area is presented by gleyic histosols, histic podzols and sod podzols (WRB, 2006) variously altered by the wildfires. Podzols are formed under forest vegetation on sandy parent material in terms of water percolation. These soils have an elivio-illuvial distribution of organomineral complexes within the profile. Histosols are characterized by the accumulation of organic matter and various compounds in thick peat horizons, and they contain more material available for burning. One important goal of our study was to establish the patterns of PAH accumulation in different soil types and to check if post-fire PAH distribution follows general soil-forming processes.

Background soils are represented by histosols and histic podzols. The histosols have the following horizons, O-H$\mathrm{Hp}-\mathrm{He}-\mathrm{Ha}-\mathrm{C}$, and a typical profile of histic podzols has $\mathrm{O}-$ A-E-EBhs-Bhs-Cs horizons. After the smoldering fire and burning out of the peat layer, the new ash horizons (Cpir) up to $5 \mathrm{~cm}$ in depth form; they are underlaid by peat charry horizons (Hpir) with $2-3 \mathrm{~cm}$ and pyrogenic muck peat horizons (Ha,pir) with $2-3 \mathrm{~cm}$ in thickness. 


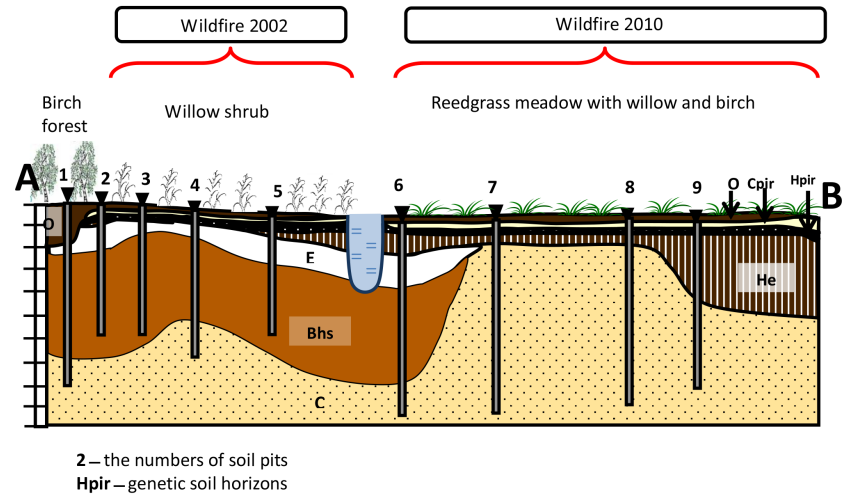

Figure 3. The profile characteristics of the investigated soils.

Post-pyrogenic histic podzols are confined to the peripheral part of peatlands; the parent material for them is bare quartz sands, and typically their profile has the following horizons: O-Cpir-Hpir-He-Ha-E-EBhs-Bhs-Bh-BCC. Post-pyrogenic gleyic histosols occupy large areas and form in the central parts of peatlands; their profile consists of O-Cpir-Hpir-He-Ha-Ch-Cs-Cg (Figs. 3, 4).

It should be pointed out that the thickness of horizons in soils affected by the fire varies, depending on the intensity of the pyrogenic impact. The depth of organogenic horizons in soil pits ranges from 10 to $30 \mathrm{~cm}$ at a distance of several meters.

\subsection{Soil sampling and laboratory analysis}

In order to study PAH accumulation in post-fire soils of different types, the areas covered with histosols (pits 7, 8, 9, 11,12 ) and sod podzols (pits $2,3,4,5,6$ ) were sampled, as well as areas with analogous background soils (pits 1,10 ) (Fig. 3).

For the comparison of PAH accumulation in soils after fires of different times, the areas affected by the fires of 2002, 2010 and 2012 were studied. The field work and sampling were conducted in 2012 and, in total, we studied 12 soil pits (Fig. 2).

Soil pits were excavated to a depth of $50-100 \mathrm{~cm}$, depending on the depth of the parent material. Detailed morphological descriptions of soil profiles were made.

For the identification of the parts of post-fire soil profiles with maximal PAH accumulation, the samples were collected from each genetic horizon and, in cases of thick horizons (more than $10 \mathrm{~cm}$ ), samples were taken at $10 \mathrm{~cm}$ intervals. Samples were stored in plastic bags and taken to the laboratory, where they were air dried, homogenized and sieved through a $0.25 \mathrm{~mm}$ sieve.

The target PAHs in this study were naphthalene, phehanthrene, chrysene, pyrene, anthracene, benz(a)anthracene, benz(a)pyrene, benz(ghi)perylene, fluorene, dibenztiophene, triphenylene, benz(e)pyrene, benz(k)fluorantene, and

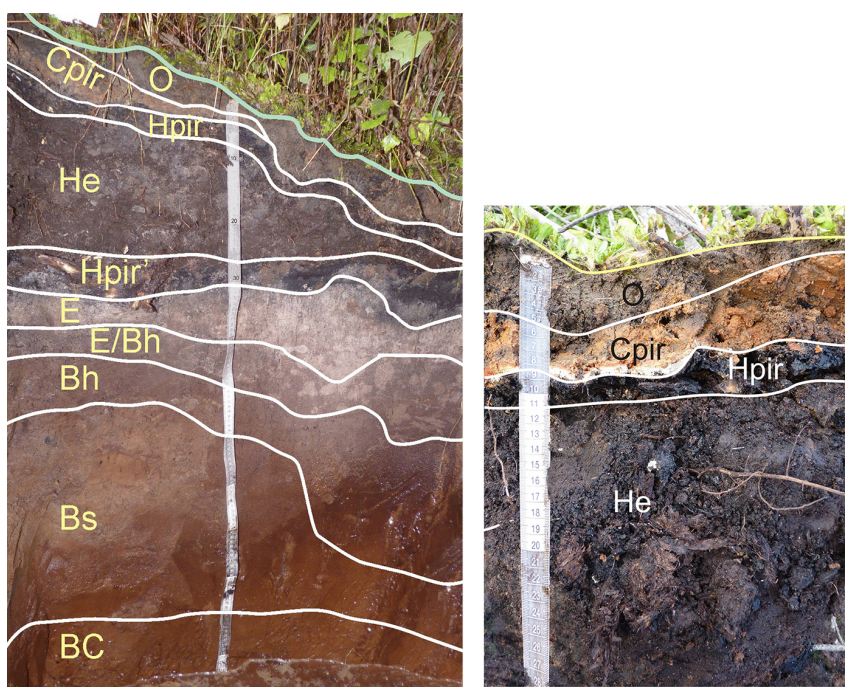

Figure 4. Soil profiles investigated. 1-post-pyrogenic histic podzol (pit 6), 2-post-pyrogenic histosol (pit 8).

coronene. This group of compounds includes PAHs from low-molecular-weight to high-molecular-weight compounds ( $2-7$ benzene rings in their structure), which are widespread in the environment.

The quantitative analysis was conducted with the specrtofluorometry method at the temperature of liquid nitrogen (Spolskii spectroscopy) (Alexeeva and Teplitskaya, 1988; Gooijer et al., 2000; Personov, 1981; Gennadiev et al., 1996).

Liquid extraction was used in the analysis. $3 \mathrm{~g}$ of air-dried soil samples were extracted with $n$ hexane $(5 \mathrm{~mL})$ at room temperature. The degree of extraction was controlled by the absence of extract luminescence under UV light. In cases of the presence of extract luminescence, the extraction was continued with $5 \mathrm{~mL}$ of $n$ hexane. This method of extraction excluded an alteration of matter, as is possible by thermal or acoustic effects and other kinds of intensive extractions.

The measurements were done on a Jobin Yvon Fluorolog3-22 spectrofluorimeter. The extract was frozen in liquid nitrogen $(77 \mathrm{~K})$. Then, the mixture of PAHs in the frozen extract was irradiated by light with optimum wavelengths for each compound, and the PAH luminescence spectra were recorded (Fig. 5).

The wavelengths of the excitation and emissions of luminescence used for the PAH identifications are given in Table 1. Spectral fractionation (identification of each hydrocarbon by the most optimum excitation and luminescence wavelengths) was used. High selectivity of the method is obtained by using a spectra selection of PAHs in multicomponent solutions by scanning the narrow excitation wave band (Alekseeva and Teplitskaya, 1981). Identification and quantitative estimations of PAHs were made by comparison of fluorescence and excitation spectra with the SRM NIST 2250a (36 PAHs mixture) reference standard solution. Limits of 
Table 1. The wavelengths of the excitation and emissions of luminescence used for the PAH identifications.

\begin{tabular}{lrr}
\hline PAH & $\begin{array}{r}\text { Excitation } \\
(\lambda, \mathrm{nm})\end{array}$ & $\begin{array}{r}\text { Emission } \\
(\lambda, \mathrm{nm})\end{array}$ \\
\hline Naphthalene & 290 & 322 \\
Phehanthrene & 293 & 346 \\
Chrysene & 269 & 361 \\
Pyrene & 352 & 372 \\
Anthracene & 253 & 378 \\
Benz(a)anthracene & 290 & 384 \\
Benz(a)pyrene & 367 & 402 \\
Benz(ghi)perylene & 367 & 419 \\
Fluorene & 288 & 302 \\
Dibenztiophene & 286 & 425 \\
Triphenylene & 287 & 462 \\
Benz(e)pyrene & 333 & 388 \\
Benz(k)fluorantene & 310 & 401 \\
Coronene & 300 & 445 \\
\hline
\end{tabular}

detection (LOD) for each PAH were $\leq 0.1 \mathrm{ng} \mathrm{g}^{-1}$. The limits of quantification (LOQ) for each PAH were $\leq 0.5 \mathrm{ng} \mathrm{g}^{-1}$. In total, 109 soil samples were analyzed for PAH concentration. The analysis was done in one replicate.

\subsection{Statistical analyses}

The results were analyzed with STATISTICA 8.0. The distribution of total PAHs in each horizon type was tested for normality with the Kolmogorov-Smirnov test, and each group included similar horizons from a similar soil type, so sample replicates were considered; the number of values in each group was from 6 to 14. The data did not follow the normal distribution at significance level $p>0.05$. So, a nonparametric Kruskal-Wallis ANOVA test was conducted for comparison of the PAH concentrations in different horizons. This test does not require the normal distribution. The differences in PAH concentrations in different horizon types were represented in categorized boxes and whisker plots. The KruskalWallis ANOVA test was also used for the statistical estimate of differences between soils affected by wildfires of different years. The upper horizons of the soils were considered in this test. All statistical tests were performed at the standard significance level $p<0.05$.

Cluster analysis was conducted to find similarities in the distributions of the individual PAH compounds. In this analysis, complete linkage was used as the amalgamation rule, and the distance metric was 1-Pearson $r$. PAHs were combined in clusters if the coefficient of correlation in every group was significant at $p<0.05$. Moreover, for each compound, the coefficient of variation was calculated.

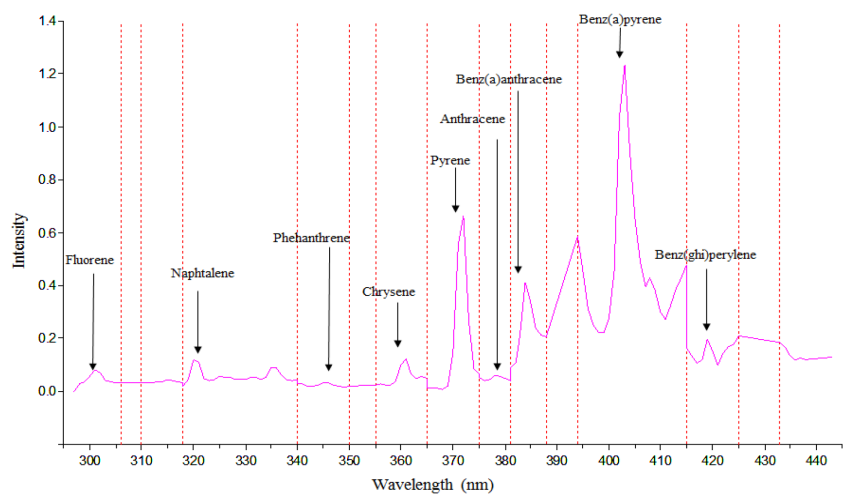

Figure 5. PAH luminescence spectra for SRM NIST 2250a.

\section{Results}

The studied soils varied highly in PAH concentrations; their total content changed from 5 to $330 \mathrm{ng} \mathrm{g}^{-1}$. In the histosols not affected by fire (pit 10), the total PAH concentrations ranged from 19.6 to $137.2 \mathrm{ng} \mathrm{g}^{-1}$ within the profile; their composition included naphthalene, banz(a)antracene and phenantrene. In the histic podzols not affected by fire (pit 1), the total PAH concentration varied from $35.4 \mathrm{ng} \mathrm{g}^{-1}$ in horizon A to $0.2 \mathrm{ng} \mathrm{g}^{-1}$ in horizon BC. Naphtalene and pyrene prevailed in this profile. In mineral horizon Bhs, only naphthalene and, sporadically, pyrene occurred. Phenanthrene and benz(a)antracene were found only in organic horizons (Table 2).

\subsection{Profile distributions of PAHs in soils of different types}

The common features of PAH distribution in post-fire histosols (pits 7, 8, 9) were the following (Fig. 6, 7, Table 3). These soils had the ash horizon Cpir at depth 2 $5 \mathrm{~cm}$, and typically at these sites they had low PAH concentrations $\left(5-8 \mathrm{ng} \mathrm{g}^{-1}\right)$. Below and above the Cpir horizon, there were two maxima of PAH concentration. The first maximum of PAH concentration was detected in surface incipient horizon $\mathrm{O}\left(20-70 \mathrm{ng} \mathrm{g}^{-1}\right)$, where mainly 23 -nuclear compounds (naphthalene, phenantrene, antracene) occurred. The second maximum was below Cpir in pyrogenic charry peat horizons (Hpir). In these horizons, the total PAH concentration reached $255 \mathrm{ng} \mathrm{g}^{-1}$. The PAH composition in these horizons was also different. Cpir horizons had predominantly low-molecular-weight compounds: 2-nuclear naphthalene and 4-nuclear pyrene. In Hpir horizons, 5-6-nuclear compounds prevailed: benz(ghi)perylene, benz(e)pyrene, benz(k)fluorantene and naphthalene occurred in lower concentrations. In underlying organic horizons, $\mathrm{He}$ and $\mathrm{Ha}$ benz(a)antracene occurred. In humic-infiltrated horizon $\mathrm{Ch}$, low PAH concentrations (up to $5-10 \mathrm{ng} \mathrm{g}^{-1}$, coefficient of variation for total PAHs $106 \%$ ) were registered, and hydrocarbons were represented only by naphthalene. 
Table 2. PAH concentrations (ng $\mathrm{g}^{-1}$ ) in soils of unburned area.

\begin{tabular}{|c|c|c|c|c|c|c|c|c|c|c|c|c|c|c|c|c|c|}
\hline 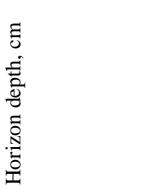 & 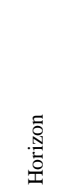 & 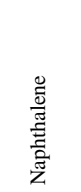 & 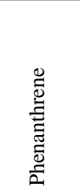 & 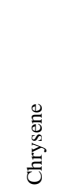 & 节 & 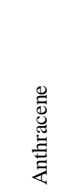 & 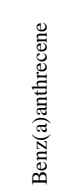 & 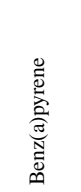 & 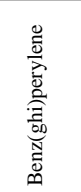 & 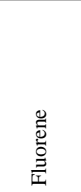 & 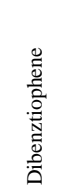 & $\frac{\stackrel{0}{0}}{\mathbb{D}}$ & 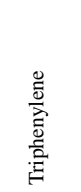 & 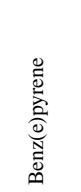 & 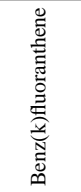 & 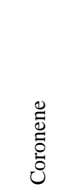 & 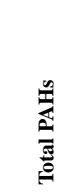 \\
\hline \multicolumn{18}{|c|}{ Histosol (pit 10) } \\
\hline $0-5$ & $\mathrm{He}$ & 11.0 & $<$ LOD & $<$ LOD & $<$ LOD & $<$ LOD & 8.6 & $<$ LOD & $<$ LOD & $<$ LOD & $<$ LOD & $<$ LOD & $<$ LOD & $<$ LOD & $<$ LOD & $<$ LOD & 19.6 \\
\hline $5-10$ & $\mathrm{He}$ & 29.1 & 36.1 & $<$ LOD & $<$ LOD & 3.4 & 11.6 & $<$ LOD & $<$ LOD & $<$ LOD & $<$ LOD & $<$ LOD & $<$ LOD & $<$ LOD & $<$ LOD & $<$ LOD & 80.2 \\
\hline $10-15$ & $\mathrm{He}$ & 40.6 & 49.3 & $<\mathrm{LOD}$ & $<$ LOD & $<$ LOD & 19.7 & $<$ LOD & $<$ LOD & $<$ LOD & $<$ LOD & $<$ LOD & $<$ LOD & $<$ LOD & $<$ LOD & $<$ LOD & 109.6 \\
\hline $15-20$ & $\mathrm{He}$ & 34.7 & 38.0 & $<\mathrm{LOD}$ & $<$ LOD & $<$ LOD & 9.6 & $<$ LOD & $<\mathrm{LOD}$ & $<$ LOD & $<$ LOD & $<$ LOD & $<$ LOD & $<$ LOD & $<$ LOD & $<$ LOD & 82.3 \\
\hline $20-30$ & $\mathrm{Ha}$ & 32.5 & $<\mathrm{LOD}$ & $<$ LOD & $<$ LOD & $<$ LOD & 16.2 & $<$ LOD & $<\mathrm{LOD}$ & $<$ LOD & $<$ LOD & $<$ LOD & $<$ LOD & $<$ LOD & $<$ LOD & $<$ LOD & 48.7 \\
\hline $30-40$ & $\mathrm{Ha}$ & 45.9 & $<\mathrm{LOD}$ & $<\mathrm{LOD}$ & $<$ LOD & $<$ LOD & 12.0 & $<$ LOD & $<$ LOD & $<$ LOD & $<$ LOD & $<$ LOD & $<$ LOD & $<$ LOD & $<$ LOD & $<$ LOD & 57.9 \\
\hline $40-50$ & $\mathrm{Ha}$ & 63.5 & 27.2 & $<\mathrm{LOD}$ & $<\mathrm{LOD}$ & $<\mathrm{LOD}$ & 46.5 & $<$ LOD & $<\mathrm{LOD}$ & $<\mathrm{LOD}$ & $<\mathrm{LOD}$ & $<\mathrm{LOD}$ & $<\mathrm{LOD}$ & $<$ LOD & $<\mathrm{LOD}$ & $<\mathrm{LOD}$ & 137.2 \\
\hline \multicolumn{18}{|c|}{ Histic podzol (pit 1) } \\
\hline $0-1$ & $\mathrm{O}$ & 8.7 & 26.7 & $<\mathrm{LOD}$ & $<$ LOD & $<$ LOD & $<$ LOD & $<$ LOD & $<\mathrm{LOD}$ & $<$ LOD & $<$ LOD & $<$ LOD & $<$ LOD & $<$ LOD & $<$ LOD & $<$ LOD & 35.4 \\
\hline $1-10$ & A & 2.7 & 6.3 & $<\mathrm{LOD}$ & $<$ LOD & 0.3 & 0.9 & $<$ LOD & $<\mathrm{LOD}$ & $<$ LOD & $<$ LOD & $<$ LOD & $<$ LOD & $<$ LOD & $<$ LOD & $<$ LOD & 10.2 \\
\hline $10-18(21)$ & A & 6.9 & 11.8 & $<\mathrm{LOD}$ & $<$ LOD & $<$ LOD & 12.1 & $<$ LOD & $<$ LOD & $<$ LOD & $<$ LOD & 0.1 & $<$ LOD & $<$ LOD & 1.3 & $<$ LOD & 32.3 \\
\hline $18(21)-26$ & $\mathrm{E}$ & 3.3 & 2.3 & $<$ LOD & $<$ LOD & $<$ LOD & 0.1 & $<$ LOD & $<\mathrm{LOD}$ & $<$ LOD & $<$ LOD & $<$ LOD & $<$ LOD & $<$ LOD & $<$ LOD & $<$ LOD & 5.6 \\
\hline $26-37(40)$ & $\mathrm{E} / \mathrm{Bhs}$ & 5.3 & $<$ LOD & $<\mathrm{LOD}$ & 0.1 & $<$ LOD & $<$ LOD & $<$ LOD & $<\mathrm{LOD}$ & $<$ LOD & $<$ LOD & $<$ LOD & $<$ LOD & $<$ LOD & $<$ LOD & $<$ LOD & 5.4 \\
\hline $37(40)-55(57)$ & Bhs & 3.0 & $<$ LOD & $<\mathrm{LOD}$ & $<$ LOD & $<$ LOD & $<$ LOD & $<$ LOD & $<$ LOD & $<$ LOD & $<$ LOD & $<$ LOD & $<$ LOD & $<$ LOD & $<$ LOD & $<$ LOD & 3.0 \\
\hline $55(57)-65$ & Cs & $<$ LOD & $<$ LOD & $<$ LOD & $<$ LOD & $<$ LOD & $<$ LOD & $<$ LOD & $<\mathrm{LOD}$ & $<$ LOD & $<$ LOD & 0.2 & $<$ LOD & $<$ LOD & $<$ LOD & $<$ LOD & 0.2 \\
\hline
\end{tabular}

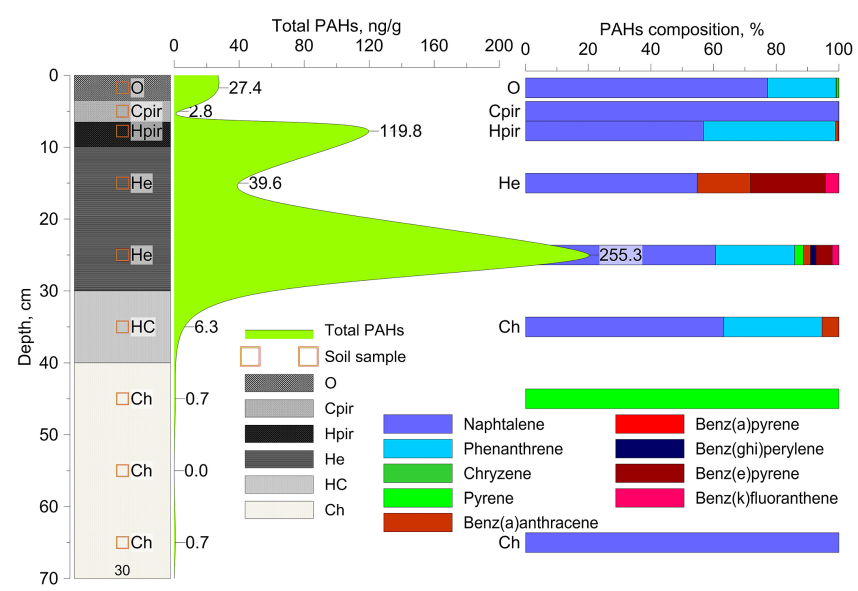

Figure 6. The profile distribution and composition of PAHs in postfire histosol in the case of a thick organogenic horizon (pit 9).

Post-fire histosols had various differences in PAH distribution. In pit 9 , the thicknesses of undestroyed peat horizons were greatest among the investigated soils (approximately $30 \mathrm{~cm}$ ), and $\mathrm{PAH}$ concentrations were also highest in this case - up to $255 \mathrm{ng} \mathrm{g}^{-1}$; moreover, PAH concentrations were relatively high within the entire depth of the organic layer. PAH composition was represented by a wide range of compounds - from low-molecular-weight to 5-6nuclear compounds - benz(ghi)perylene, benz(e)pyrene and benz $(\mathrm{k})$ fluorantene. In cases of more complete combustion of peat (pits 8 and 7) where mineral material was present from 10 to $14 \mathrm{~cm}$ in depth, the total PAH concentrations were much lower - from 5 to $25 \mathrm{ng} \mathrm{g}^{-1}$ (Fig. 7).

It may be noted that total PAH concentrations in residual peat horizons of post-fire histosols changed from 10 to $255 \mathrm{ng} \mathrm{g}^{-1}$, with coefficients of variation in the He horizon of

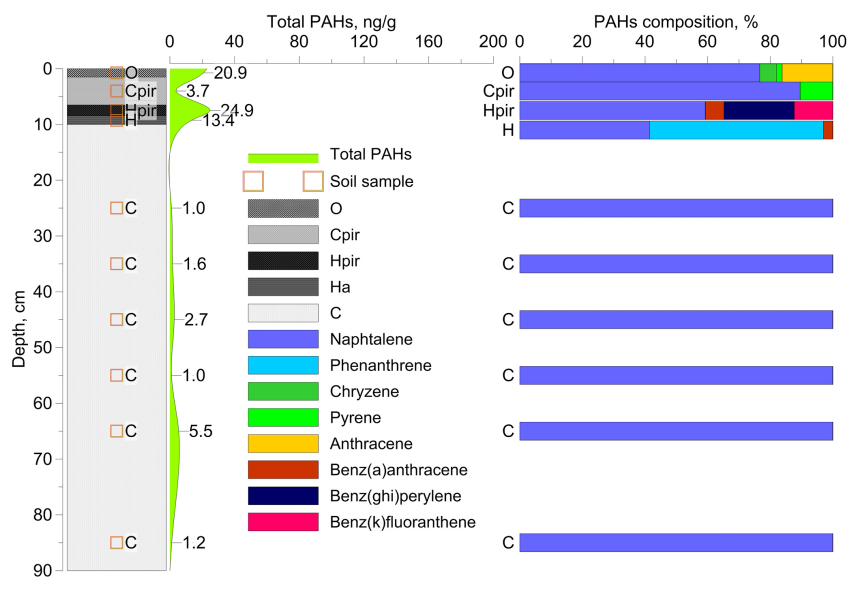

Figure 7. The profile distribution and composition of PAHs in postfire histosol in the case of almost complete burning out of the organic layer (pit 8).

118 and $155 \%$. At sites with more complete combustion of peat, PAH molecules accumulated in smaller amounts (10$20 \mathrm{ng} \mathrm{g}^{-1}$ ). In cases of incomplete burning, their concentrations in peat horizons were greater than $200 \mathrm{ng} \mathrm{g}^{-1}$.

The Kruskal-Wallis ANOVA test indicated significant differences in total PAH concentrations between different horizon types of histosols. Significant differences were detected between mineral $\mathrm{C}$ and post-fire Hpir horizons, and between mineral $\mathrm{C}$ and organogenic $\mathrm{H}$ horizons at $p<0.05$. In addition, mineral horizons $(\mathrm{Ch}, \mathrm{Cs}, \mathrm{C}$ ) had the smallest ranges and mean values, and organogenic horizons (Hpir, He, Ha) had the greatest ranges and mean values (Fig. 8).

Sod podzols and histic podzols (pits 4, 5, 2, 3, and 6) subjected to wildfires were slightly different in levels of PAH accumulation. Also, there were two maxima of total 
Table 3. PAH concentrations $\left(\mathrm{ng}^{-1}\right)$ in post-fire soils.

\begin{tabular}{|c|c|c|c|c|c|c|c|c|c|c|c|c|c|c|c|c|c|}
\hline 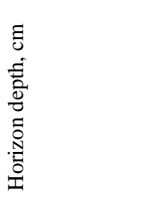 & 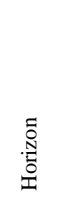 & 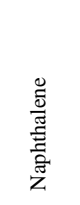 & 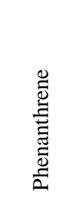 & 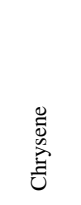 & 总 & 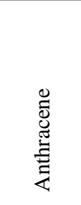 & 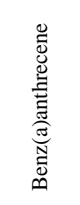 & 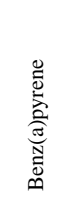 & 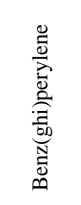 & $\begin{array}{l}\stackrel{0}{ \pm ٍ \Xi ~} \\
\stackrel{\Xi}{\Xi}\end{array}$ & 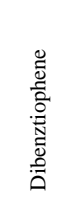 & 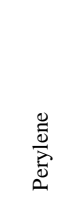 & 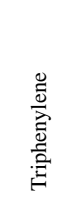 & 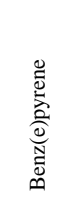 & 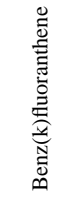 & 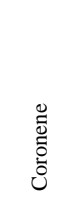 & \\
\hline \multicolumn{18}{|c|}{ Histosol (Pit 11) } \\
\hline $0-0.5$ & $\mathrm{O}$ & 55.5 & 19.4 & $<$ LOD & 1.1 & 0.3 & $<$ LOD & $<$ LOD & $<$ LOD & $<$ LOD & 0.1 & $<$ LOD & $<$ LOD & $<$ LOD & $<$ LOD & $<$ LOD & 76.5 \\
\hline $0.5-2(4)$ & Cpir & 8.3 & $<$ LOD & $<$ LOD & 0.1 & 0.1 & $<$ LOD & $<\mathrm{LOD}$ & $<$ LOD & $<\mathrm{LOD}$ & $<$ LOD & $<\mathrm{LOD}$ & $<$ LOD & $<$ LOD & $<\mathrm{LOD}$ & $<$ LOD & 8.5 \\
\hline $2(4)-7$ & Hpir & 0.9 & 0.6 & $<$ LOD & $<$ LOD & $<$ LOD & 0.1 & $<\mathrm{LOD}$ & 2.6 & $<\mathrm{LOD}$ & $<$ LOD & $<\mathrm{LOD}$ & $<$ LOD & 2.4 & $<$ LOD & 0.2 & 6.7 \\
\hline $7-8$ & $\mathrm{He}$ & 1.4 & 0.4 & $<\mathrm{LOD}$ & $<$ LOD & $<$ LOD & 0.3 & $<$ LOD & 3.8 & $<$ LOD & $<$ LOD & $<$ LOD & $<\mathrm{LOD}$ & $<$ LOD & $<$ LOD & $<\mathrm{LOD}$ & 5.9 \\
\hline $8-14$ & $\mathrm{He}$ & $<$ LOD & $<$ LOD & $<$ LOD & $<$ LOD & $<$ LOD & $<$ LOD & $<$ LOD & $<$ LOD & $<$ LOD & $<$ LOD & $<$ LOD & $<$ LOD & $<$ LOD & $<$ LOD & 0.3 & 0.3 \\
\hline $14-26$ & $\mathrm{Ch}$ & 1.2 & $<$ LOD & $<$ LOD & 0.2 & $<$ LOD & 0.1 & $<\mathrm{LOD}$ & $<\mathrm{LOD}$ & $<\mathrm{LOD}$ & $<$ LOD & $<\mathrm{LOD}$ & $<$ LOD & $<\mathrm{LOD}$ & $<$ LOD & $<$ LOD & 1.5 \\
\hline $26-38$ & Cs & 1.2 & 1.5 & $<$ LOD & 0.2 & $<$ LOD & 6.5 & $<\mathrm{LOD}$ & $<\mathrm{LOD}$ & $<\mathrm{LOD}$ & $<$ LOD & 0.2 & $<$ LOD & $<\mathrm{LOD}$ & $<\mathrm{LOD}$ & $<\mathrm{LOD}$ & 9.7 \\
\hline $38-50$ & $\mathrm{Cs}$ & $<$ LOD & $<$ LOD & $<$ LOD & $<$ LOD & $<\mathrm{LOD}$ & $<\mathrm{LOD}$ & $<\mathrm{LOD}$ & $<\mathrm{LOD}$ & $<\mathrm{LOD}$ & $<\mathrm{LOD}$ & $<\mathrm{LOD}$ & $<$ LOD & $<\mathrm{LOD}$ & $<\mathrm{LOD}$ & $<$ LOD & 0.0 \\
\hline $50-60$ & $\mathrm{C}$ & 1.8 & $<$ LOD & $<$ LOD & 0.2 & $<$ LOD & 0.1 & $<\mathrm{LOD}$ & $<$ LOD & $<\mathrm{LOD}$ & $<\mathrm{LOD}$ & $<\mathrm{LOD}$ & $<$ LOD & $<$ LOD & $<\mathrm{LOD}$ & $<\mathrm{LOD}$ & 2.1 \\
\hline $60-70$ & $\mathrm{C}$ & 1.0 & $<$ LOD & $<$ LOD & 0.2 & $<\mathrm{LOD}$ & $<\mathrm{LOD}$ & $<\mathrm{LOD}$ & $<\mathrm{LOD}$ & $<\mathrm{LOD}$ & $<\mathrm{LOD}$ & $<\mathrm{LOD}$ & $<$ LOD & $<$ LOD & $<\mathrm{LOD}$ & $<\mathrm{LOD}$ & 1.2 \\
\hline $70-80$ & $\mathrm{C}$ & $<$ LOD & $<$ LOD & $<$ LOD & $<$ LOD & $<$ LOD & $<$ LOD & $<$ LOD & $<$ LOD & $<$ LOD & $<$ LOD & $<$ LOD & $<$ LOD & $<$ LOD & $<$ LOD & $<$ LOD & 0.0 \\
\hline $80-90$ & $\mathrm{C}$ & 0.5 & $<$ LOD & $<$ LOD & 0.2 & $<$ LOD & $<$ LOD & $<$ LOD & $<\mathrm{LOD}$ & $<$ LOD & $<$ LOD & $<$ LOD & $<$ LOD & $<$ LOD & $<$ LOD & $<$ LOD & 0.7 \\
\hline \multicolumn{18}{|c|}{ Sod podzol (pit 5) } \\
\hline $0-2(3)$ & $\mathrm{O}$ & 9.4 & $<$ LOD & $<\mathrm{LOD}$ & $<$ LOD & $<$ LOD & $<$ LOD & $<$ LOD & $<$ LOD & $<\mathrm{LOD}$ & $<\mathrm{LOD}$ & 0.3 & $<$ LOD & $<\mathrm{LOD}$ & $<$ LOD & $<$ LOD & 9.7 \\
\hline $2(3)-3(4)$ & Cpir & 11.2 & 38.8 & 24.0 & 0.8 & 62.7 & $<$ LOD & $<\mathrm{LOD}$ & $<\mathrm{LOD}$ & $<\mathrm{LOD}$ & $<$ LOD & $<\mathrm{LOD}$ & $<$ LOD & 8.9 & $<$ LOD & $<\mathrm{LOD}$ & 146.4 \\
\hline $3(4)-6(7)$ & Apir & 48.2 & $<$ LOD & $<$ LOD & 0.8 & $<$ LOD & $<$ LOD & $<$ LOD & 0.6 & $<$ LOD & $<$ LOD & $<$ LOD & $<$ LOD & $<$ LOD & $<$ LOD & $<$ LOD & 49.6 \\
\hline $6(7)-10(14)$ & $\mathrm{E}$ & 5.5 & $<$ LOD & $<$ LOD & $<$ LOD & $<$ LOD & $<\mathrm{LOD}$ & $<\mathrm{LOD}$ & $<\mathrm{LOD}$ & $<\mathrm{LOD}$ & $<\mathrm{LOD}$ & $<\mathrm{LOD}$ & $<$ LOD & $<\mathrm{LOD}$ & $<$ LOD & $<\mathrm{LOD}$ & 5.5 \\
\hline $10(14)-20(22)$ & $\mathrm{Ha}$ & 6.1 & $<$ LOD & $<$ LOD & $<$ LOD & $<$ LOD & 0.5 & $<$ LOD & $<\mathrm{LOD}$ & $<$ LOD & $<$ LOD & $<$ LOD & $<$ LOD & 3.7 & $<$ LOD & $<$ LOD & 10.3 \\
\hline $20(22)-30(33)$ & $\mathrm{Ha}$ & 4.4 & $<$ LOD & $<$ LOD & $<$ LOD & $<$ LOD & $<$ LOD & $<$ LOD & 0.2 & $<$ LOD & $<$ LOD & $<$ LOD & $<$ LOD & $<$ LOD & $<$ LOD & $<$ LOD & 4.5 \\
\hline $30(33)-40$ & Bhs & 3.6 & $<$ LOD & $<$ LOD & $<$ LOD & $<$ LOD & $<$ LOD & $<\mathrm{LOD}$ & $<$ LOD & $<$ LOD & $<$ LOD & $<$ LOD & $<$ LOD & $<$ LOD & $<$ LOD & $<$ LOD & 3.6 \\
\hline $40-50$ & Bhs & 1.4 & $<$ LOD & $<$ LOD & 0.2 & $<$ LOD & $<$ LOD & $<\mathrm{LOD}$ & $<$ LOD & $<\mathrm{LOD}$ & $<$ LOD & $<\mathrm{LOD}$ & $<$ LOD & $<$ LOD & $<$ LOD & $<\mathrm{LOD}$ & 1.6 \\
\hline $50-60$ & Bhs & 3.7 & $<$ LOD & $<\mathrm{LOD}$ & $<$ LOD & $<$ LOD & $<$ LOD & $<$ LOD & $<$ LOD & $<$ LOD & $<$ LOD & $<$ LOD & $<\mathrm{LOD}$ & $<$ LOD & $<$ LOD & $<\mathrm{LOD}$ & 3.7 \\
\hline $60-70$ & $\mathrm{BC}$ & 1.0 & $<$ LOD & $<$ LOD & $<$ LOD & $<$ LOD & 0.9 & $<$ LOD & $<$ LOD & $<$ LOD & $<$ LOD & $<$ LOD & $<$ LOD & $<$ LOD & $<$ LOD & $<$ LOD & 1.9 \\
\hline \multicolumn{18}{|c|}{ Sod podzol (pit 8) } \\
\hline $0-1(1.5)$ & $\mathrm{O}$ & 36.2 & OD & $<$ LOD & $<$ LOD & $<\mathrm{LOD}$ & 5.5 & LOD & $<\mathrm{LOD}$ & $<\mathrm{LOD}$ & $<$ LOD & $<\mathrm{LOD}$ & $<\mathrm{LOD}$ & $<$ LOD & $<$ LOD & $<\mathrm{LOD}$ & 41.7 \\
\hline $1(1.5)-2(3)$ & Apir & 10.7 & $<$ LOD & $<\mathrm{LOD}$ & 0.1 & 0.5 & $<$ LOD & $<$ LOD & $<$ LOD & $<$ LOD & $<$ LOD & $<$ LOD & $<$ LOD & $<$ LOD & $<$ LOD & $<$ LOD & 11.3 \\
\hline $4(5)-7$ & $\mathrm{H}$ & 40.2 & 11.6 & $<$ LOD & 8.2 & $<$ LOD & $<\mathrm{LOD}$ & $<\mathrm{LOD}$ & 4.2 & $<\mathrm{LOD}$ & $<\mathrm{LOD}$ & $<\mathrm{LOD}$ & $<$ LOD & $<\mathrm{LOD}$ & $<\mathrm{LOD}$ & $<\mathrm{LOD}$ & 64.2 \\
\hline $2(3)-7$ & Hpir & 42.4 & $<$ LOD & $<\mathrm{LOD}$ & $<$ LOD & $<$ LOD & $<$ LOD & $<$ LOD & $<$ LOD & $<$ LOD & $<$ LOD & $<$ LOD & $<$ LOD & $<$ LOD & $<$ LOD & $<$ LOD & 42.4 \\
\hline $7-9$ & $\mathrm{Ha}$ & 5.4 & $<$ LOD & $<$ LOD & $<$ LOD & 1.7 & 0.7 & $<$ LOD & $<$ LOD & $<$ LOD & $<$ LOD & $<\mathrm{LOD}$ & $<$ LOD & $<$ LOD & $<$ LOD & $<$ LOD & 7.8 \\
\hline $9-14$ & $\mathrm{Ha}$ & 1.0 & $<$ LOD & $<$ LOD & $<$ LOD & $<$ LOD & $<$ LOD & $<$ LOD & $<$ LOD & $<$ LOD & $<$ LOD & $<$ LOD & $<$ LOD & $<$ LOD & $<$ LOD & $<$ LOD & 1.0 \\
\hline $14-25$ & $\mathrm{E}$ & 1.3 & $<$ LOD & $<$ LOD & $<$ LOD & $<$ LOD & 0.1 & $<$ LOD & $<$ LOD & $<$ LOD & $<$ LOD & $<$ LOD & $<$ LOD & $<$ LOD & $<\mathrm{LOD}$ & $<$ LOD & 1.5 \\
\hline $25-30$ & $\mathrm{E}$ & $<$ LOD & $<$ LOD & $<\mathrm{LOD}$ & $<$ LOD & $<$ LOD & $<$ LOD & $<$ LOD & $<\mathrm{LOD}$ & $<$ LOD & $<$ LOD & $<$ LOD & $<\mathrm{LOD}$ & $<$ LOD & $<$ LOD & $<\mathrm{LOD}$ & 0.0 \\
\hline $30-40$ & Bs & 1.2 & $<$ LOD & $<\mathrm{LOD}$ & $<$ LOD & $<$ LOD & $<$ LOD & $<\mathrm{LOD}$ & $<\mathrm{LOD}$ & $<\mathrm{LOD}$ & $<$ LOD & $<\mathrm{LOD}$ & $<$ LOD & $<$ LOD & $<$ LOD & $<\mathrm{LOD}$ & 1.2 \\
\hline $40-50$ & Bs & 0.7 & $<$ LOD & $<$ LOD & $<$ LOD & $<$ LOD & $<$ LOD & $<$ LOD & $<$ LOD & $<$ LOD & $<$ LOD & $<$ LOD & $<$ LOD & $<$ LOD & $<$ LOD & $<$ LOD & 0.7 \\
\hline \multicolumn{18}{|c|}{ Sod podzol (Pit 9) } \\
\hline $0-1(2)$ & $\mathrm{O}$ & 2.4 & $<$ LOD & $<\mathrm{LOD}$ & $<$ LOD & $<$ LOD & $<$ LOD & $<$ LOD & 0.7 & $<\mathrm{LOD}$ & $<\mathrm{LOD}$ & $<\mathrm{LOD}$ & $<\mathrm{LOD}$ & $<\mathrm{LOD}$ & $<$ LOD & $<\mathrm{LOD}$ & 3.1 \\
\hline $1(2)-4$ & Cpir & 11.5 & $<$ LOD & $<\mathrm{LOD}$ & 0.3 & 0.4 & $<\mathrm{LOD}$ & $<$ LOD & $<\mathrm{LOD}$ & $<\mathrm{LOD}$ & $<$ LOD & $<\mathrm{LOD}$ & $<\mathrm{LOD}$ & $<$ LOD & $<\mathrm{LOD}$ & $<\mathrm{LOD}$ & 12.3 \\
\hline $4-5$ & Apir & 30.2 & 22.6 & $<\mathrm{LOD}$ & $<$ LOD & 13.2 & 3.5 & $<$ LOD & $<\mathrm{LOD}$ & $<\mathrm{LOD}$ & $<\mathrm{LOD}$ & $<\mathrm{LOD}$ & $<\mathrm{LOD}$ & $<\mathrm{LOD}$ & $<$ LOD & $<\mathrm{LOD}$ & 69.5 \\
\hline $5-7$ & A & 10.7 & 7.8 & $<\mathrm{LOD}$ & 1.2 & $<$ LOD & $<$ LOD & $<$ LOD & $<$ LOD & $<$ LOD & $<$ LOD & $<$ LOD & $<\mathrm{LOD}$ & $<$ LOD & $<$ LOD & $<$ LOD & 19.7 \\
\hline $7-12$ & $\mathrm{E}$ & 1.6 & 2.6 & $<\mathrm{LOD}$ & $<\mathrm{LOD}$ & $<$ LOD & $<\mathrm{LOD}$ & $<\mathrm{LOD}$ & $<\mathrm{LOD}$ & $<\mathrm{LOD}$ & $<\mathrm{LOD}$ & $<\mathrm{LOD}$ & $<$ LOD & $<\mathrm{LOD}$ & $<\mathrm{LOD}$ & $<\mathrm{LOD}$ & 4.2 \\
\hline $12-20$ & EB & 1.5 & $<$ LOD & $<$ LOD & $<$ LOD & $<$ LOD & $<$ LOD & $<$ LOD & $<$ LOD & $<$ LOD & $<\mathrm{LOD}$ & $<$ LOD & $<$ LOD & $<$ LOD & $<\mathrm{LOD}$ & $<\mathrm{LOD}$ & 1.5 \\
\hline $20-30$ & Bs & 3.3 & $<$ LOD & $<$ LOD & $<$ LOD & $<$ LOD & $<$ LOD & $<$ LOD & $<\mathrm{LOD}$ & $<$ LOD & $<$ LOD & $<$ LOD & $<$ LOD & $<$ LOD & $<$ LOD & $<$ LOD & 3.3 \\
\hline $30-40$ & Bs & 12.2 & $<$ LOD & $<$ LOD & $<$ LOD & $<$ LOD & $<$ LOD & $<$ LOD & $<$ LOD & $<$ LOD & $<$ LOD & $<$ LOD & $<$ LOD & $<$ LOD & $<$ LOD & $<$ LOD & 12.2 \\
\hline $40-50$ & B & 11.4 & $<\mathrm{LOD}$ & $<$ LOD & $<$ LOD & $<$ LOD & $<\mathrm{LOD}$ & $<\mathrm{LOD}$ & $<\mathrm{LOD}$ & $<\mathrm{LOD}$ & $<\mathrm{LOD}$ & $<\mathrm{LOD}$ & $<$ LOD & $<\mathrm{LOD}$ & $<\mathrm{LOD}$ & $<\mathrm{LOD}$ & 11.4 \\
\hline
\end{tabular}

PAH concentrations in these soils. The first maximum was most frequently confined to pyrogenic organogenic soil horizons (Hepir, Hapir, Apir, He, Ha); these contained up to $40-60 \mathrm{ng} \mathrm{g}^{-1}$. The composition of hydrocarbons in these horizons and the contribution of separate components to PAH associations differed slightly. In most cases, PAHs were represented by naphthalene, anthracene, and pyrene. In particular cases, high-molecular-weight PAHs (perylene, benz(ghi)perylene, benz(k)fluoranthene, baez(a)anthracene) appeared (Figs. 9, 10, Table 3).

Furthermore, occasionally, Cpir and post-pyrogenic $\mathrm{O}$ horizons of pits 4 and 5 , closer $(50 \mathrm{~m})$ to the burned area of 2010, contained up to $150 \mathrm{ng} \mathrm{g}^{-1} \mathrm{PAH}$. These sites were af- fected by the fire of 2002, perhaps because they were directly nearby the site of a recent burned area (Fig. 3).

At a distance from the site of the last fire, the Cpir horizons (pits 2 and 3) (Table 3), formed in the fire of 2002, contain PAHs in low amounts - up to $2-3 \mathrm{ng} \mathrm{g}^{-1}$, in some cases - to $10-12 \mathrm{ng} \mathrm{g}^{-1}$. Naphthalene prevailed among hydrocarbons, while in soil pit 3, 3-4-nuclear PAHs - chrysene, pyrene, anthracene - were added. The post-fire surface incipient $\mathrm{O}$ horizons in these soils contained $3-40 \mathrm{ng} \mathrm{g}^{-1}$ PAHs. PAH composition was comprised of naphtalenes and traces of banz(a)anthracene, perylene, and benz(ghi)perylene.

In contrast to histosols, the sod podzols are characterized by eluvio-illuvial translocation of hydrocarbons. For 
Table 4. PAH concentrations (ng $\mathrm{g}^{-1}$ ) in soils of recently burned areas (2012).

\begin{tabular}{|c|c|c|c|c|c|c|c|c|c|c|c|c|c|c|c|c|c|}
\hline 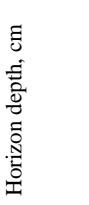 & 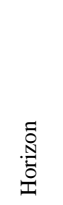 & 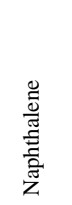 & 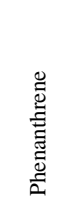 & 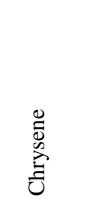 & 营 & 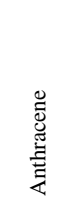 & 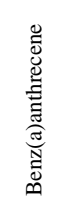 & 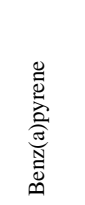 & 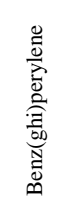 & $\begin{array}{l}\stackrel{\Xi}{0} \\
\stackrel{\Xi}{\Xi}\end{array}$ & 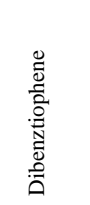 & $\frac{\ddot{D}}{\frac{0}{2}}$ & 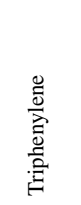 & 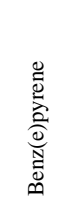 & 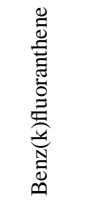 & 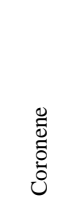 & 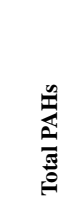 \\
\hline \multicolumn{18}{|c|}{ Histosol, the plot of recently burned areas (2012; pit 13). } \\
\hline $0-3$ & $\mathrm{O}$ & 9.2 & $<$ LOD & 0.2 & 0.3 & $<$ LOD & $<$ LOD & $<$ LOD & $<$ LOD & $<$ LOD & $<\mathrm{LOD}$ & $<$ LOD & $<$ LOD & $<$ LOD & 0.2 & $<$ LOD & 9.8 \\
\hline $3-5(6)$ & Hpir & 285.8 & $<$ LOD & $<\mathrm{LOD}$ & $<$ LOD & 46.8 & $<$ LOD & $<\mathrm{LOD}$ & $<$ LOD & $<\mathrm{LOD}$ & $<\mathrm{LOD}$ & $<$ LOD & $<$ LOD & $<$ LOD & $<$ LOD & $<$ LOD & 332.6 \\
\hline $7(9)-16$ & $\mathrm{He}$ & 68.5 & 21.3 & $<\mathrm{LOD}$ & $<\mathrm{LOD}$ & $<\mathrm{LOD}$ & $<$ LOD & $<\mathrm{LOD}$ & 6.2 & $<\mathrm{LOD}$ & $<\mathrm{LOD}$ & $<$ LOD & $<\mathrm{LOD}$ & 10.0 & $<$ LOD & $<\mathrm{LOD}$ & 106.0 \\
\hline \multicolumn{18}{|c|}{ Histosol, the plot adjacent to the burned area (2012; pit 14). } \\
\hline $0-1$ & Hpir & 31.4 & 21.0 & $<$ LOD & 8.3 & $<$ LOD & 12.5 & 3.1 & $<$ LOD & $<\mathrm{LOD}$ & $<\mathrm{LOD}$ & $<$ LOD & $<$ LOD & $<$ LOD & $<$ LOD & $<$ LOD & 76.3 \\
\hline $1-10$ & $\mathrm{He}$ & 39.8 & $<$ LOD & $<$ LOD & $<\mathrm{LOD}$ & $<$ LOD & 77.9 & $<$ LOD & $<$ LOD & $<$ LOD & $<\mathrm{LOD}$ & $<$ LOD & $<$ LOD & $<$ LOD & $<$ LOD & $<$ LOD & 117.6 \\
\hline $10-11$ & Cpir & 70.3 & 8.5 & $<\mathrm{LOD}$ & 2.8 & $<$ LOD & 3.5 & $<$ LOD & 0.2 & $<$ LOD & $<\mathrm{LOD}$ & $<$ LOD & $<$ LOD & $<$ LOD & $<$ LOD & $<$ LOD & 85.3 \\
\hline $11-11.5$ & Hpir & 15.2 & $<$ LOD & 0.1 & $<$ LOD & $<$ LOD & $<$ LOD & $<$ LOD & 3.7 & $<\mathrm{LOD}$ & $<$ LOD & $<$ LOD & $<$ LOD & $<$ LOD & $<$ LOD & $<$ LOD & 19.0 \\
\hline $11.5-20$ & $\mathrm{He}$ & 120.5 & $<\mathrm{LOD}$ & $<\mathrm{LOD}$ & $<\mathrm{LOD}$ & 15.6 & $<$ LOD & $<\mathrm{LOD}$ & $<$ LOD & $<\mathrm{LOD}$ & $<\mathrm{LOD}$ & $<$ LOD & $<$ LOD & $<$ LOD & $<$ LOD & $<$ LOD & 136.0 \\
\hline $20-30$ & $\mathrm{He}$ & 66.6 & $<$ LOD & $<\mathrm{LOD}$ & $<$ LOD & $<$ LOD & $<$ LOD & $<\mathrm{LOD}$ & $<$ LOD & $<$ LOD & $<\mathrm{LOD}$ & $<$ LOD & $<$ LOD & $<$ LOD & $<$ LOD & $<$ LOD & 66.6 \\
\hline $30-40$ & $\mathrm{He}$ & 60.1 & $<$ LOD & $<\mathrm{LOD}$ & 1.9 & $<$ LOD & 3.1 & $<$ LOD & 1.3 & $<\mathrm{LOD}$ & $<\mathrm{LOD}$ & $<\mathrm{LOD}$ & $<$ LOD & $<$ LOD & $<$ LOD & $<$ LOD & 66.3 \\
\hline $40-45$ & $\mathrm{He}$ & 114.3 & $<$ LOD & $<\mathrm{LOD}$ & $<$ LOD & $<$ LOD & 1.3 & 0.2 & $<$ LOD & $<$ LOD & $<\mathrm{LOD}$ & $<$ LOD & $<$ LOD & $<$ LOD & $<$ LOD & $<\mathrm{LOD}$ & 115.8 \\
\hline
\end{tabular}

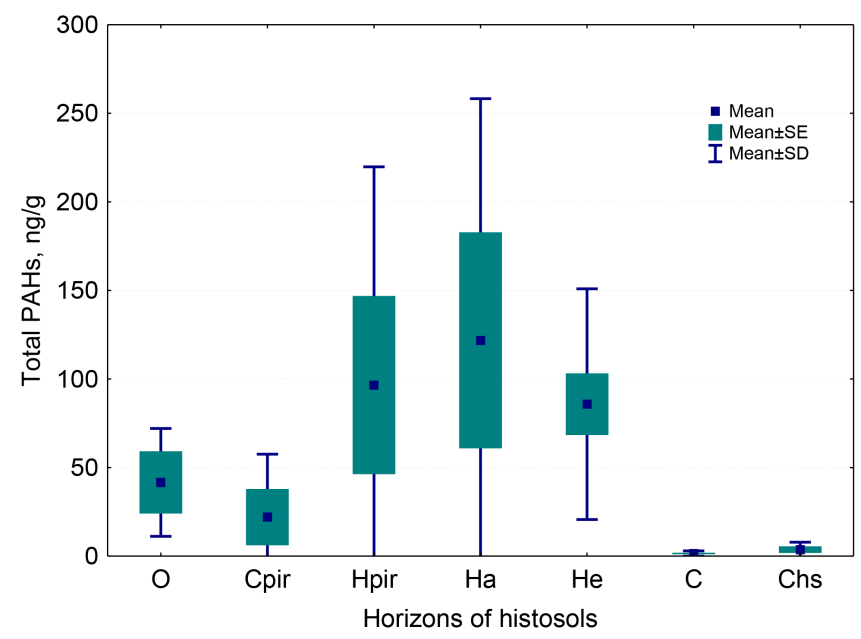

Figure 8. The categorized box and whisker plot for total PAHs in different horizons of histosols (pits 7, 8, and 9). The number of sample replicates: O-3, Cpir-5, Hpir-6, He-14, Ha-5, Chs-5, and C-12. Horizons $\mathrm{Cs}$ and $\mathrm{Ch}$ were combined for this analysis, indicated by Chs.

instance, eluvial horizons E contained 0.7 to $5 \mathrm{ng} \mathrm{g}^{-1}$ PAHs with a coefficient of variation of $98 \%$ and, in some cases, hydrocarbons were not detected. The PAHs were represented in these horizons only by naphthalene and trace amounts of phenanthrene and pyrene. The upper parts of illuvial Bhs horizons had total concentrations in amounts of up to $12 \mathrm{ng} \mathrm{g}^{-1}$, and PAH composition included mainly naphthalene, but benz(a)antracene, fluorene, benz(e)pyrene and benz(k)fluorantene occurred in some cases. So, in contrast to background sod podzols, the post-fire soils tended to have some migration of high-molecular weight compounds to illuvial soil horizons (Fig. 10).

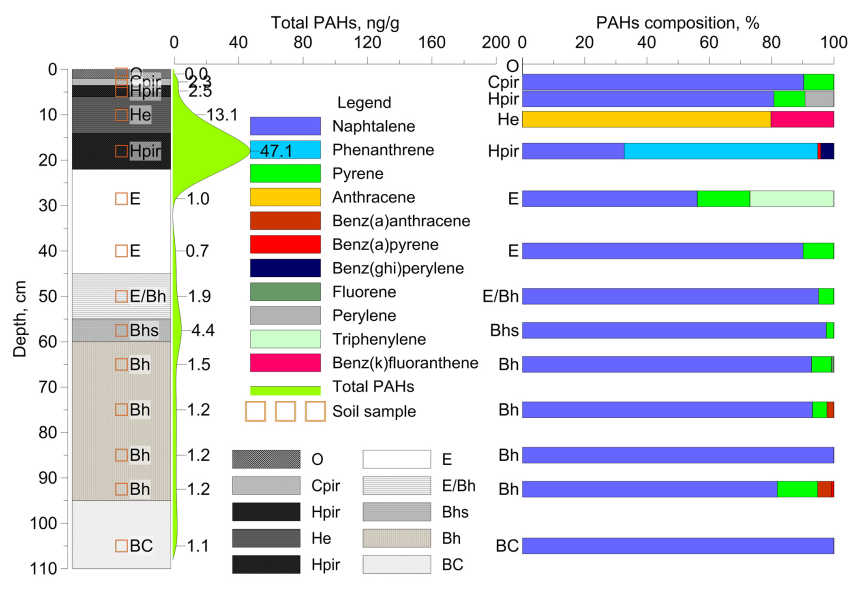

Figure 9. The profile distribution and PAH composition in postpyrogenic histic podzols, wildfire 2010 (pit 6).

As opposed to histosols O, Cpir and Apir, horizons of histic and sod podzols had high levels of PAHs that exceeded the range in pyrogenic organogenic horizons (Fig. 11). In podzols, significant differences in PAH concentrations were identified between the $\mathrm{A}$ and $\mathrm{E}$ and between the $\mathrm{A}$ and $\mathrm{B}$ horizons $(p<0.05)$.

In the investigated soils, PAHs tended to form groups different in their distribution, which was confirmed by cluster analysis (Fig. 12). PAHs were combined in clusters if the coefficient of correlation in every group was significant at $p<0.05$. Low-molecular-weight 2 - and 3-nuclear compounds had similar distributions in both histosols and podzols throughout the whole profile. The 5-6-nuclear compounds had similar distributions, and were detected only in organogenic horizons Hpir, $\mathrm{Ha}$, and $\mathrm{He}$.

Among the samples, the coefficient of variation was high for high-molecular-weight compounds, especially in 


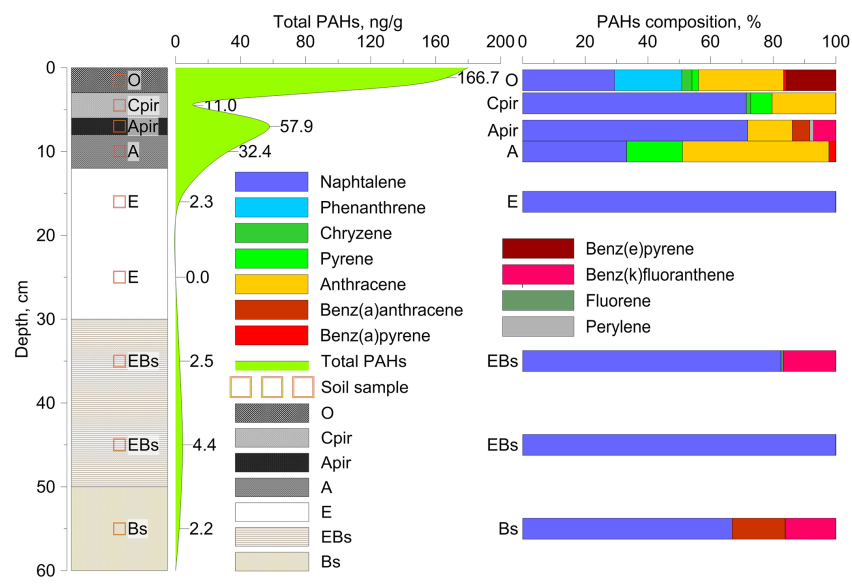

Figure 10. The profile distribution and PAH composition in postpyrogenic histic podzols from the wildfire of 2002 (pit 5).

Hpir horizons, for benz(a)pyrene ( $283 \%$ ), benz(ghi)perylene $(283 \%)$, benz(k)fluorantene (437\%), and chrysene (195\%). The variation of low-molecular-weight compounds was lower; in $\mathrm{H}$ horizons, coefficients of variation for naphthalene were 131 and $126 \%$ for phenanthrene.

\subsection{The PAH accumulation in soils of burned sites of different times}

Certain differences in the intensity of PAH accumulation could be noted in soils of different age burned sites. On the site burned in 2012, the post-fire histosol within the burned area (pit 12) and the histosol situated nearby the burned site and containing charred peat layers from previous fires (pit 11) were studied. In the soil of this most recently burned site, the total PAH concentrations were the highest among all the sites studied. Here, PAH concentrations were $330 \mathrm{ng} \mathrm{g}^{-1}$, and were confined to charry peat horizon Hpir (Table 4). The PAH composition was represented by naphthalene and anthracene. In a deeper horizon $\mathrm{He}$ of postfire histosol without charry material high-molecular weight, compounds benz(ghi)perylene and benz(e)pyrene occurred. The same compounds were detected in soil adjacent to the burned area, but in a lesser amount; the total PAH concentration did not exceed $140 \mathrm{ng} \mathrm{g}^{-1}$. In comparison with the histosols of the 2 year post-burn area (pits 8 and 9), this site is characterized by a higher PAH concentration.

In the case of sod podzols affected by the fires of 2002 and 2010 (pits 5 and 6), the pyrogenic organogenic horizons had similar PAH concentrations (Fig. 9, 10). The differences in the intensities of eluvio-illuvial processes were not detected.

The Kruskal-Wallis ANOVA test indicated significant differences in PAH concentrations, depending on the number of years following the fire. Soils affected by the fire of 2012 differed significantly from years 2002 and 2010, but the differ-

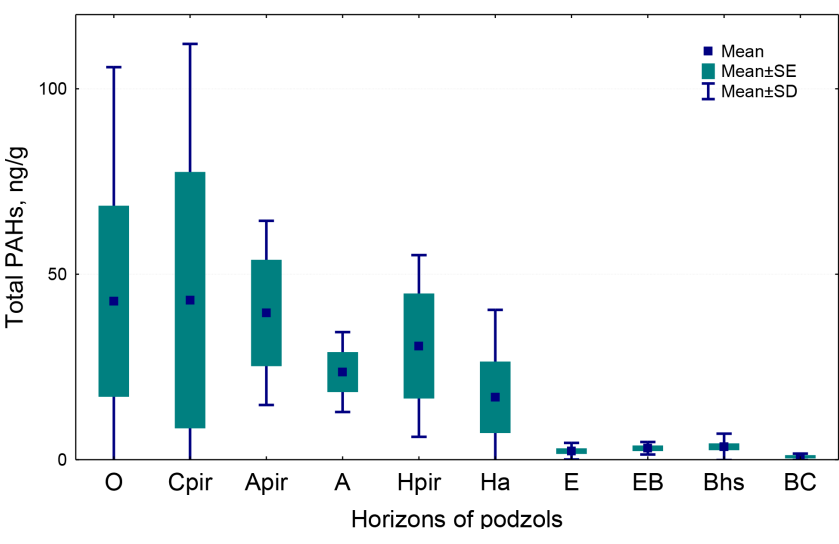

Figure 11. The categorized box and whisker plot for total PAHs in different horizons of podzols (pits 2, 3, 4, 5, and 6). The number of sample replicates: O-6; Cpir-4 Apir-3; A-3; Hpir-3; Ha-6; E-9; EB-5; Bhs-15; BC-4. Horizons Bh, Bs, and Bhs were combined for this analysis into Bhs.

ences between 2002 and 2010 were not detected $(p<0.05)$. Generally, the organogenic horizons from the fire in $2012 \mathrm{had}$ higher PAH concentrations (Fig. 13). In the surface ash horizons Cpir of burned areas from 2002, the tendencies of PAH concentrations decreasing were not detected.

\section{Discussion}

\subsection{Profile distribution of PAHs in soils of different types}

The data on PAH concentration and composition in soils not affected by fires corresponded to results obtained for soils of other territories distant from PAH sources (Gennadiev and Tsibart, 2013; Wilcke, 2007; Rovinskii, 1988; Gabov et al., 2007; Krasnopeeva, 2008).

The production of PAHs in Hpir horizons of histosols could be the result of the structure of the smoldering front. These horizons correspond to the pyrolysis front, where wide various volatile compounds and hydrocarbons, including PAHs, are formed. Above this, horizon oxidation and ash formation processes take place with no PAH formation (Rein, 2013). Under the conditions of smoldering and low oxygen access, PAHs may actively form in the combustion zone (Blomqvist et al., 2006; Jenkins et al., 1996; Mastral et al., 1999). Moreover, the intense PAH formation during the long-duration heating has been shown in the literature (Gonzalez-Vila et al., 1991); so, in cases of incomplete burning of peat horizons, the conditions for high PAH production tend to be present. Also, possibly, during the fire, not only the Hpir horizon, but also the entire peat layer was subjected to heating, and PAH formation could take place in organogenic horizons at all depths. 


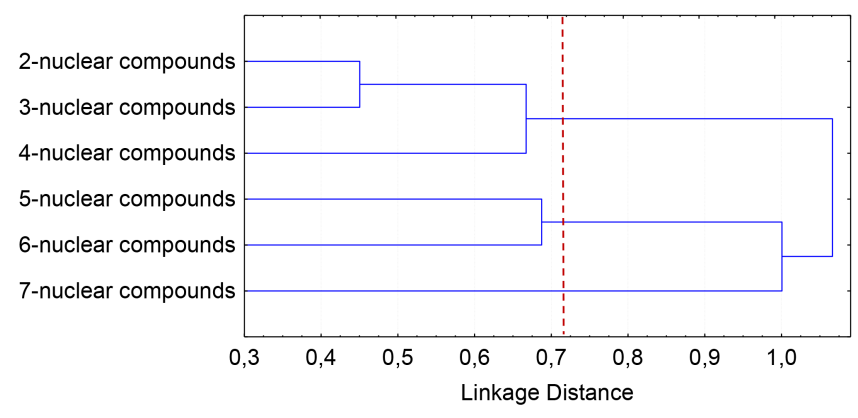

Figure 12. The cluster analysis (complete linkage; 1-Pearson $r$ ) for PAH groups in the investigated soil samples. To the left of the red line, the correlation coefficient is statistically significant $(p<0.05)$.

In addition, a possible factor causing different PAH concentrations in peat horizons was the amount of peat remaining after the burning. The greater the residual mass of peat, the greater the sorption of PAHs that was observed to take place. At the same time, allowing for the high variability of absolute PAH concentrations in residual peat horizons, it could be surmised that they tend to accumulate 5-6-nuclear compounds, which could indicate processes of peat combustion. Vane et al. (2013) also pointed out the presence of lowand high-molecular PAHs in soils after peat fire. However, 5-6-nuclear compounds (benz(ghi)perylene, benz(a)pyrene, benz(e)pyrene, benz(k)fluotanthene) could be considered the indication group marking the peat combustion.

The soils with very shallow (up to several $\mathrm{cm}$ ) peat horizons remaining following fire had low PAH concentrations due to the fact that almost all organic matter had been totally burned out. High coefficients of variation of PAHs in residual peat horizons could be caused by different durations and depths of heating of these horizons at different sites. The depth of changes in soils differs depending on the fire intensity and location characteristics (varying moisture content, microrelief and peat thickness) (Efremova and Efremov, 2006; Grishin et al., 2013).

In the surface incipient horizon $\mathrm{O}\left(20-70 \mathrm{ng} \mathrm{g}^{-1}\right)$, where mainly 2-3-nuclear compounds (naphthalene, phenantrene, antracene) occurred, these compounds were presumably sorbed by new organogenic horizons from the atmosphere. Sorption is one of the leading processes in soil involving PAHs (Trapido, 1999; Wicke, 2000), and the factors determining the rate of sorption are the content of the organic matter and the presence of clay minerals (Maliszewska-Kordybach, 2005; Maliszewska-Kordybach et al., 2010). These were quite high in the newly formed organic horizons.

The PAH concentrations in pyrogenic organogenic horizons (Hepir, Hapir, Apir, He, Ha) of podzols were lower than in analogous horizons of histosols, as these soils had a lesser amount of available organic material for burning and $\mathrm{PAH}$ formation.

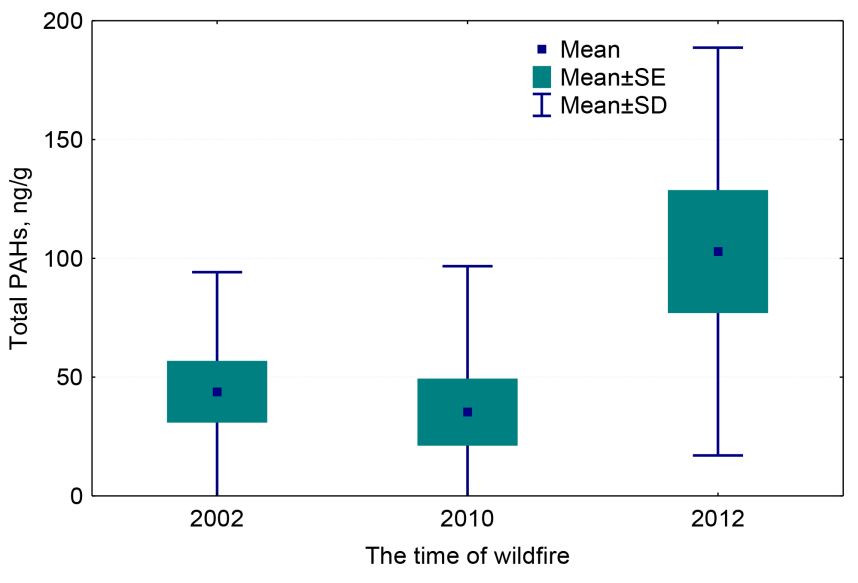

Figure 13. The categorized box and whisker plot for total PAHs in horizons Hpir, Apir, O and Cpir of soils affected by the fires of 2002, 2010, and 2012 (pits 2, 3, 4, 5, 6, 7, 8, 9, 11, and 12). The number of sample replicates: 2002 year $-15,2010$ year $-19,2012$ year -11 .

Also, in comparison with histosols, Cpir and post-fire O horizons of podzols had rather high PAH concentrations. These findings could be explained by the fact that these sites were affected by fire in 2002, but were also directly nearby the site of a burned area of 2010 (Fig. 3). These horizons have a sandy loam texture (Zaidel'man et al., 2006); so, they have a high sorption capacity. Presumably, the accumulation of low-molecular-weight compounds formed during the last fire could take place at these sites.

Also, probably, as the accumulation of heavy PAHs takes place in situ in char and ash horizons, PAHs are translocated across them to the atmosphere, because these horizons act as filters of gases. The accumulation of low-molecular-weight PAHs could be explained by atmospheric input; these compounds are released into the atmosphere with other combustion products, where they are translocated and then deposited at other plots (Rein, 2013).

In contrast to histosols, the sod podzols were characterized by eluvio-illuvial translocation of hydrocarbons, which was also found in the study of Gabov (2007); so, PAH distribution follows the soil-forming processes typical of the wet climates of taiga biomes. The increased migration of PAHs after the fire was, probably, caused by vegetation destruction during the fire and following intense percolation through the soil profile.

\subsection{The PAH accumulation in soils of burned sites of different times}

The histosols affected by the fire in 2012 had a higher PAH concentration in comparison with the histosols after the fire of 2010. On the one hand, it caused arriving PAHs, especially low-molecular-weight compounds, to possibly degrade after the fire. On the other hand, in the present case, the organic horizons of the most recently burned site were not 
completely burned out, which favors the intense sorption and accumulation of polycyclic aromatic hydrocarbons in these soils, but it is worth mentioning that the time of the pyrogenic PAH presence in the soil could differ, depending on the conditions. Thus, even on a geological scale, the elevated PAH concentration in the deposits of the Cretaceous and Jurassic periods could be explained by ubiquitous occurrence of wildfire (Killops and Massourd, 1992; Marynowski et al., 2011; Belcher, 2006). However, according to GarciaFalcoan (2006), the concentrations in burned organo-mineral soils remained high only during the first 3 months after the fire and, then, PAHs were reduced by soil processes including degradation and migration.

In the case of sod podzols from the 2002 fire, the concentrations of PAHs could also be influenced by the 2010 fires; therefore, trends of PAH degradation with time were not so prominent.

Considering the soils of drained peatlands, it is necessary to emphasize that, in both post-fire sod podzols and histosols, the total PAH concentrations could be rather high - up to hundreds of $\mathrm{ng} \mathrm{g}^{-1}$. These peculiarities differ from the features found in our previous studies - in Polistovo Natural Reserve (Pskov region, Russia), where post-fire organogenic peat soils have higher PAH concentrations in comparison with sod-podzol soils (Gennadiev and Tsibart, 2013). Moreover, in the undrained soils, the total PAH concentrations did not exceed $150 \mathrm{ng} \mathrm{g}^{-1}$. Presumably, in the Shatura district, the deep changes in the hydrologic regime occurred after the drainage and the level of groundwater decreased. In these cases, fire affected the soil cover of the area to a great extent, and new horizons having different capacities for PAH accumulation formed. Also, after the catastrophic influence of the fire, the heterogeneity of the soil cover increased, resulting in highly contrasting soil profiles and spatial PAH distribution.

However, it should be noted that even under conditions of catastrophic fires on the drained peatlands in the studied cases, the PAH concentrations, especially highmolecular-weight compounds, were relatively low: for example, benz(a)pyrene concentrations did not exceed $3 \mathrm{ng} \mathrm{g}^{-1}$.

The findings from this study correspond to some previously reported results in the literature. Vane et al. (2013) note that PAH concentrations in peat soils after fire are lower than the amount dangerous for biota. Also, there is no evidence that amounts of PAHs accumulated after the fire are dangerous to humans, as they are lower than hazardous levels determined for soils, but the variation of high-molecular-weight PAHs in soil horizons was high, as these compounds do not occur in all horizons, and their formation is a more complicated process in comparison with low-molecular PAHs.

\section{Conclusions}

The conclusions of our study are the following:
1. The wildfires on the drained peatlands caused the change in the morphological properties of soils; new soil horizons Cpir, O, Hpir, and Hapir were formed with different accumulation rates of PAHs. The highest PAHs concentrations were seen in charry peat Hpir horizons and in post-fire incipient $\mathrm{O}$ horizons.

2. Post-fire histosols and histic podzols differed in their PAH distribution. In both cases, the highest PAH concentrations occurred in the organogenic post-fire horizons, but in cases of histic podzols, their slight accumulation of PAHs in illuvial horizons was possible.

3. 5-6-nuclear compounds were formed in pyrogenic horizons Ha,pir and He,pir. Their production was facilitated in smoldering processes due to a lack of oxygen; this group could be considered an indicator group of peat combustion. 2-3-nuclear PAHs occurred within the whole profile; in sod podzols and histic podzols, their migration to illuvial horizons was observed. In the cases we studied, PAH composition in pyrogenic horizons did not change in different soil types.

4. The trends of higher PAH accumulation in soils were observed in cases of incomplete burning out of peat horizons. PAH sorption in upper horizons of soils near the sites of the most recent fires was observed.

Acknowledgements. This work was supported by the Russian Geographical Society and by the Russian Foundation for Basic Research, project 12-05-31314.

Edited by: P. Pereira

\section{References}

Agarwal, T.: Concentration level, pattern and toxic potential of PAHs in traffic soil of Delhi, India, J. Haz. Mater., 171, 894-900, 2009.

Alexeeva, T. A. and Teplitskaya, T. A.: Spectrofluorometric methods of polycyclic aromatic analysis in natural and technogenic medias, Leningrad, 215 pp., 1981.

Baek, S. O., Field, R. A., Goldstone, M. E., Kirk, P. W., Lester, J. N., and Perry, R.: A review of atmospheric polycyclic aromatic hydrocarbons: sources, fate and behavior, Water Air Soil Pollut., 60, 279-300, 1991.

Bandowe, B., Meusela, H., Huang, R., Hod, K., Cao, J., Hoffmann, T., and Wilcke, W.: PM2.5-bound oxygenated PAHs, nitro-PAHs and parent-PAHs from the atmosphere of a Chinese megacity: Seasonal variation, sources and cancer risk assessment, Sci. Total Environ., 473/474, 77-87, 2014.

Belcher, C. M.: Impacts and wildfires - an analysis of the K-T event, Biological processes associated with impact events, 221243, 2006 
Belis, C. A., Offenthaler, I., and Weiss, P.: Semivolatiles in the forest environment: the case of PAHs, Plant Ecophysiology, Organic Xenobiotics and Plants, Part 1, 47-73, 2001.

Betha, R., Pradani, M., Lestari, P., Joshi, U., Reid, J., and Balasubramanian, R.: Chemical speciation of trace metals emitted from Indonesian peat fires for health risk assessment, Atmos. Res., 122, 571-578, 2013.

Blake, D., Hinwood, A. L., and Horwitz, P.: Peat fires and air quality: Volatile organic compounds and particulates, Chemosphere, 76, 419-423, 2009.

Blomqvist, P., Persson, B., and Simonson, M.: Fire emissions of organics into the atmosphere, Fire Technol., 43, 213-231, 2007.

Bojakowska, I. and Sokołowska, G.: Polycyclic aromatic hydrocarbons in materials of burned peatlands, Polish J. Environ.1 Stud., 12, 401-408, 2003.

Cai, Q., Mo, C., Wu, Q., Katsoyiannis, A., and Zeng, Q.: The status of soil contamination by semivolatile organic chemicals (SVOCs) in China: A review, Sci. Total Environ., 389, 209-224, 2008.

Cooijer, C., Ariese F., and Hofstraat, J. W.: Shpol'skii Spectroscopy and Other Site-Selection Methods, Applications in Environmental Analysis, Bioanalytical Chemistry and Chemical Physics, John Wiley \& Sons, NY, 552 pp., 2000.

di Folco, M. and Kirkpatrick, J.: Topographic variation in burninginduced loss of carbon from organic soils in Tasmanian moorlands, Catena, 87, 216-225, 2011.

Donkelaar, A., Martin, R., Levy, R., da Silva, A., Krzyzanowski, M., Chubarova, N., Semutnikova, E., and Cohen, A.: Satellite-based estimates of ground-level fine particulate matter during extreme events: A case study of the Moscow fires in 2010, Atmos. Environ., 45, 6225-6232, 2011.

Dymov, A. A., Dubrovsky, Yu. A., and Gabov, D. N.: Pyrogenic changes in iron-illuvial podzols in the middle taiga of the Komi Republic, Eurasian Soil Science, 47, 47-56, 2014.

Efremova, T. T. and Efremov, S. P.: Pyrogenic transformation of organic matter in soils of forest bogs, Eurasian Soil Sci., 39, 12971305, 2006.

Fitzpatrick, E. M., Jones, J. M., Pourkashanian, M., Ross, A. B., Williams, A., and Bartle, K. D.: Mechanistic aspects of soot formation from the combustion of pine wood, Energy and Fuels, 22, 3771-3778, 2008.

Freeman, D. J. and Cattell, F. C. R..: Woodburning as a source of atmospheric polycyclic aromatic hydrocarbons, Environ. Sci. Technol., 24, 1581-1585, 1990.

Gabov, D. N., Beznosikov, V. A., and Kondratenok, B. M.: Polycyclic aromatic hydrocarbons in background podzolic and gleyic peat-podzolic soils, Eurasian Soil Sci., 40, 256-264, 2007.

Garcia-Falcoan, M. S., Soto-Gonzaalez, B., and Simal-Gaandara, J.: Evolution of the concentrations of polycyclic aromatic hydrocarbons in burnt woodland soils, Environ. Sci. Technol., 40, 759763, 2006.

Gennadiev, A. N. and Tsibart, A. S.: Pyrogenic polycyclic aromatic hydrocarbons in soils of reserved and anthropogenically modified areas: factors and features of accumulation, Eurasian Soil Sci., 46, 28-36, 2013.

Gennadiev, A. N., Pikovskii, Yu. I., Florovskaya, V. N., Alexeeva T. A., Kozin, I. S., Ogloblina, A. I., Ramenskaya, M. E., Teplitskaya, T. A., and Shurubor, E. I: Geochemistry of Polycyclic
Aromatic Hydrocarbons in Rocks in Soils, Moscow University publ., Moscow, 188 pp., 1996.

Gonzalez-Vila, F., Lopez, J., Martin, F., and del Rio, J.: Determination in soils of PAH produced by combustion of biomass under different conditions, Fresenius J. Anal Chem., 339, 750-753, 1991.

Grishin, A. M., Zima, V. P., and Kasymov, D. P.: On the deepening mechanism of the site of peat combustion, J. Engin. Phys. Thermophys., 86, 996-1001, 2013.

Guo, W., Pei, Y, Yang, Z., and Chen, H.: Historical changes in polycyclic aromatic hydrocarbons (PAHs) input in Lake Baiyangdian related to regional socio-economic development, J. Haz. Mater., 187, 441-449, 2011.

Haritash, A. K. and Kaushik, C. P.: Biodegradation aspects of Polycyclic Aromatic Hydrocarbons (PAHs): A review, J. Haz. Mater., 169, 1-15, 2009.

Hartford, R. A. and Frandsen, W. H.: When It's Hot, It's Hot... Or Maybe It's Not! (Surface Flaming May Not Portend Extensive Soil Heating), Internat. J. Wildland Fire, 2, 139-144, 1992.

Jenkins, B., Jones, A. D., Turn, S. Q., and Williams, R. B.: Emission factors for polycyclic aromatic hydrocarbons from biomass burning, Environ. Sci. Technol., 30, 2462-2469, 1996.

Kakareka, S. V., Kukharchic, T. I., Zanevslaya, L. A., Kurman, P. V., Chuduk, V. N., and Chomich, V. S.: Modeling and assessment of PAHs emissions during wildfires in peatlands, in: Natural resources, 10, The institute on natural resources usage and ecology of Belorussian National Academy, Belorussia, 58-62, 2004.

Khan, A., Ishaq, M., and Khan, M.: Effect of vehicle exhaust on the quantity of polycyclic aromatic hydrocarbons (PAHs) in soil, Environ. Monit. Assess., 137, 363-369, 2008.

Killops, S. D. and Massourd, M. S.: Polycyclic aromatic hydrocarbons of pyrolytic origin in ancient sediments: evidence for Jurassic vegetation fires, Org. Geochem., 1, 1-7, 1992.

Krasnopeeva, A.: Natural bituminoids in soils of the forest zone: Luminescence diagnostics and content levels (Satino Research Station, Moscow State University), Eurasian Soil Sci., 41, 12821293, 2008.

Kudravtseva, N. L.: Geostructural location and conditions of peat bogs and deep lakes nourishment in Meshchera downfold and on the Oka-Tsna bar, in: The Aspects of ground water and engineergeological processes studying, Russia, Moscow, 74-84, 1973.

Kwon, H. and Choi, S.: Polycyclic aromatic hydrocarbons (PAHs) in soils from a multi-industrial city, South Korea, Sci. Total Environ., 470/471, 1494-1501, 2014.

Maioli, O. L., Knoppers, B. A., and Azevedo, D. A.: Sources, distribution and variability of hydrocarbons in total atmospheric suspended particulates of two Brazilian areas influenced by sugarcane burning, J. Atmos. Chem., 64, 159-178, 2009.

Maisto, G., Nicola, F., Iovieno, P., Prati, M. V., and Alfani, A.: PAHs and trace elements in volcanic urban and natural soils, Geoderma, 136, 20-27, 2006.

Maliszewska-Kordybach, B.: Dissipation of polycyclic aromatic hydrocarbons in freshly contaminated soils - the effect of soil physicochemical properties and aging, Water Air Soil Pollut., 168, 113-128, 2005.

Maliszewska-Kordybach, B., Klimkowicz-Pawlas, A., Smreczak, B., and Stuczyński, T.: Relationship between soil concentrations of PAHs and their regional emission indices, Water Air Soil Pollu., 213, 319-330, 2010. 
Marynowski, L., Scott, A. C., Zatoń, M., Parent, H., and Garrido, A. C: First multi-proxy record of Jurassic wildfires from Gondwana: Evidence from the Middle Jurassic of the Neuquén Basin, Argentina, Palaeogeogr. Palaeocl., 299, 129-136, 2011.

Masclet, P., Cachier, H., Liousse, C., and Wortham, H.: Emissions of polycyclic aromatic hydrocarbons by savanna fires, J. Atmos. Chem., 22, 41-54, 1995.

Mastral, A. and Callen, M.: A Review on Polycyclic Aromatic Hydrocarbon (PAH) Emissions from Energy Generation, Environ. Sci. Technol., 34, 3051-3057, 2000.

Mastral, A., Callean, M., and Garcia T.: Polycyclic aromatic hydrocarbons and organic matter associated to particulate matter emitted from atmospheric fluidized bed coal combustion, Environ. Sci. Technol., 33, 3177-3184, 1999.

Medeiros, P. and Simoneit, B.: Source profiles of organic compounds emitted upon combustion of green vegetation from temperate climate forests, Environ. Sci. Technol., 42, 8310-8316, 2008.

Mu, L., Peng, L., Cao, J., He, Q., Li, F., Zhang, J., Liu, X., and Bai, $\mathrm{H}$.: Emissions of polycyclic aromatic hydrocarbons from coking industries in China, Particuology, 11, 86-93, 2013.

Nakajima, D., Nagame, S., Kuramochi, H., Sugita, K., Kageyama, S., Shiozaki, T., Takemura, T., Shiraishi, F., and Goto, S.: Polycyclic aromatic hydrocarbon generation behavior in the process of carbonization of wood, Bull Environ Contam Toxicol., 79, 221-225, 2007.

Nussbaumer, T.: Combustion and co-combustion of biomass: fundamentals, technologies, and primary measures for emission reduction, Energy Fuels, 17, 1510-1521, 2003.

Personov, R. I.: Site Selection Spectroscopy of Organic Molecules in Solutions and its Application, J. Luminescence, 24/25, p. 475, 1981.

Radojevic, M.: Chemistry of forest fires and regional haze with emphasis on Southeast Asia, Pure appl. Geophys, 160, 157-187, 2003.

Ramdahl, T. and Bechler, G.: Characterization of polynuclear aromatic hydrocarbon derivatives in emissions from wood and cereal straw combustion, Anal. Chimica Ac., 1982, 144, 83-91, 1982.

Rein, G.: Smouldering Fires and Natural Fuels, Fire Phenomena and the Earth System: An Interdisciplinary Guide to Fire Science, Chapter 2, 15-34, 2013.

Rein, G., Cleaver, N., Ashton, C., Pironi, P., and Torero, J. L.: The severity of smouldering peat fires and damage to the forest soil, Catena, 74, 304-309, 2008.

Rovinskii, F. Ya., Teplitskaya, T. A., and Alexeeva, T. A.: Background monitoring of polycyclic aromatic hydrocarbons, Leningrad, 224 pp., 1988.

Schauer, J., Kleeman, M., Cass, G., and Simoneit, B.: Measurement of emissions from air pollution sources. 3. C1-C29 organic compounds from fireplace combustion of wood, Environ. Sci. Technol., 35, 1716-1728, 2001.

Shvidenko, A. Z., Shchepashchenko, D. G., Vaganov, E. A., Sukhinin, A. I., Maksyutov, Sh. Sh., McCallum, I., and Lakyda, I. P.: Impact of Wildfire in Russia between 1998-2010 on Ecosystems and the Global Carbon Budget, Doklady Earth Sciences, 441, Part 2, 1678-1682, 2011.

Simakin, G. Ya.: Peat resources of Moscow Region, Moscow, Russia, 74 pp., 1958.
Simoneit, B.: A review of biomarker compounds as source indicators and tracers for air pollution, Environ. Sci. and Pollut. Res., 6, 159-169, 1999.

Sushkova, I. V.: The description of the cover disturbances taken place on the former peat-mining territories in Shaturskiy district of Moscow region and further development prognosis of vegetation in the area, Bulletin of Moscow State Regional University, Series "Natural sciences', 2, 43-46, 2008.

Timashev, A.: Shatura power plant, Moscow-Leningrad, Russia, 128 pp., 1932.

Trapido, M.: Polycyclic aromatic hydrocarbons in Estonian soil: contamination and profiles, Environ. Pollut., 105, 67-74, 1999.

Tsibart, A. S. and Gennadiev, A. N.: Polycyclic Aromatic Hydrocarbons in Soils: Sources, behavior, and Indication Significance (A Review), Eurasian Soil Sci., 46, 728-741, 2013.

Vane, C. H., Rawlins, B. G., Kim, A. W., Moss-Hayes, V., Kendrick, C. P., and Leng, M. J.: Sedimentary transport and fate of polycyclic aromatic hydrocarbons (PAH) from managed burning of moorland vegetation on a blanket peat, South Yorkshire, UK, Sci. Total Environ., 449, 81-94, 2013.

Vergnoux, A., Malleret, L., Asia, L., Doumenq, P., and Theraulaz, F.: Impact of forest fires on PAH level and distribution in soils, Environ. Res., 111, 193-198, 2011.

Wetlands of Moscow Region, Natural, economic and historicalcultural aspects, Wetland International-Russia Programme, KMK, Scientific press, Moscow, Russia, 89 pp., 2008.

Wilcke, W.: Polycyclic aromatic hydrocarbons (PAHs) in soil - a review, J. Plant Nutr. Soil Sci., 163, 229-248, 2000.

Wilcke, W.: Global patterns of polycyclic aromatic hydrocarbons (PAHs) in soil, Geoderma, 141, 157-166, 2007.

WRB: World Reference base for soil resources 2006, 2nd edition, World Soil Resources Reports, No 103, Food and Agriculture Organization of the United Nations, Rome, 2006

Yuan, H., Tao, S., Li, B., Lang, C., Cao, J., and Coveney, R. M.: Emission and outflow of polycyclic aromatic hydrocarbons from wildfires in China, Atmospheric Environment, 42, 6828-6835, 2008.

Zaccone, C., Rein, G., D’Orazio, V., Hadden, R. M., Belcher, C. M., and Miano, T. M.: Smouldering fire signatures in peat and their implications for palaeoenvironmental reconstructions, Geochim. Cosmochim. Ac., 137, 134-146, 2014.

Zaidel'man, F. R. and Romanov, S. V.: Ecological-hydrothermal assessment of pyrogenic soils of cutover peatlands, Euras. Soil Sci., 40, 82-92, 2007.

Zaidel'man, F. R., Bannikov, M. V., and Shvarov, A. P.: Properties and fertility of pyrogenic formations on burnt drained peaty soils, Euras. Soil Sci., 32, 1032-1039, 1999.

Zaidel'man, F. R., Morozova, D. I., and Shvarov, A. P.: Changes in the properties of pyrogenic formations and vegetation on burnt previously drained peat soils of poles'ie landscapes, Euras. Soil Sci., 36, 1159-1167, 2003.

Zaidel'man, F. R., Morozova, D. I., Shvarov, A. P., and Batrak, M. V.: Vegetation and pedogenesis on pyrogenic substrates of former peat soils, Euras. Soil Sci., 39, 12-20, 2006.

Zonov, N. T. and Konstantinovitch, A. E.: Geologic structure, mineral resources and ground waters of Egorievskii, Shaturskii, Korobovskii and Kurovskii Districts of Moscow Region, Transactions of Moscow geological survey bureau, 11, Moscow, Russia, 1932. 